Génét. Sél. Evol., 1985, 17 (4), 499-538

\title{
Efficacité génétique du transfert d'embryons dans les noyaux de sélection chez les bovins laitiers
}

\author{
J.J. COLLEAU \\ I.N.R.A., Station de Génétique quantitative et appliquée \\ Centre de Recherches Zootechniques, F 78350 Jouy-en-Josas
}

\begin{abstract}
Résumé
Les techniques actuelles de superovulation, de collecte et de transfert des embryons chez les bovins permettent théoriquement la mise en place de nouveaux programmes de sélection. Dans cet article, on aborde l'étude génétique d'un mode particulier de noyaux de sélection, utilisant le transfert d'œufs et en vue de la sélection d'un seul caractère : la production laitière. Ces noyaux ont pour fonction et nouveauté essentielles la production systématique de très jeunes mères à taureaux connues sur une lactation suivant 2 collectes d'embryons, qui commence en moyenne après la naissance des produits, de manière à raccourcir l'intervalle de génération sur les voies femelles. La méthode de testage des mâles sur descendance et la méthode de production des pères à taureaux sont identiques à celles utilisées à l'heure actuelle. Elles utilisent notamment le contrôle laitier national.

L'évolution génétique des veaux nés dans le noyau est comparée à celle des veaux issus d'accouplements raisonnés dans le cadre d'un schéma classique, avec la contrainte que le nombre annuel des mâles testés et que les procédures de sélection sur la voie père-fils sont les mêmes dans les 2 cas. Le raccourcissement de l'intervalle de génération dans le noyau et l'utilisation de taureaux bien connus sont des facteurs favorables à une accélération du progrès génétique en comparaison d'un schéma classique de référence très efficace (progrès génétique annuel $=0,23$ écart-type génétique). Cette accélération est fonction de la taille du noyau et des paramètres techniques du transfert (nombre et taux de survie des embryons). La supériorité est de l'ordre de 20 p. 100 quand la taille du noyau est comparable à l'effectif du nombre de taureaux à tester, pour les paramètres techniques actuels (nombre d'embryons par récolte $=4-5 ;$ taux de survie des embryons $=40-60$ p. 100). La sensibilité de ces schémas à ces paramètres est moins importante que celle des schémas proposés par Nicholas \& SMith (MOET) en 1983.

Dans le court terme, les valeurs relatives du progrès génétique ne sont guère modifiées quand ont tient compte dans les calculs de facteurs habituellement négligés (déséquilibre de linkage induit par la sélection; supériorité génétique vraie de reproducteurs apparentés sélectionnés dans une population de taille limitée). En ce qui concerne le long terme, le problème de l'évolution vraie de la variance génétique, notamment dans les schémas de transfert, n'a pas été réellement abordé.
\end{abstract}

Mots clés : Sélection, variance génétique, consanguinité, noyaux, transfert d'embryons, bovins laitiers. 


\author{
Summary \\ Genetic improvement by embryo transfer within selection nuclei \\ in dairy cattle
}

With current techniques to induce superovulation and collect and transfer embryos in cattle, the development of new selection schemes is theoretically possible. A genetic study of a special type of selection nuclei using embryo transfer and intended for selection on one trait only, i.e. milk production, is described in this paper. The systematic production of very young bull dams evaluated from their 1st lactation following 2 embryo recoveries and starting, on an average, after birth of the offspring so as to reduce the generation interval along the female paths, represents the main role and novelty of these nuclei. The methods used for the progeny testing of the males and production of bull sires were similar to those applied at present, involving in particular the national milk recording data.

The genetic change in calves born within the nuclei was compared to that of calves from planned matings within a conventional scheme. However, there were 2 constraints, i.e. use of the same annual number of tested males and the same selection procedure along the sire-son path in both cases. Reduction of the generation interval within the nuclei and use of well-known bulls favored an acceleration of the annual genetic gain, compared to a very efficient conventional selection scheme $(0.23$ genetic standard deviation). This acceleration depended on nucleus size and on some technical parameters of transfer such as embryo number and survival rate. There was about a 20 p. 100 improvement when nucleus size was the same as the annual number of bulls to be tested (present parameters : number of embryos per recovery $=4-5$; embryo survival rate $=40-60$ p. 100). The sensitivity of these programs to the above parameters was lower than that of the programs proposed by NicHOLAS \& SMITH (MOET).

On a short-term basis, the relative values of genetic progress were not modified much when factors such as linkage disequilibria induced by selection and true genetic superiority of apparented breeding animals selected within a population of restricted size (usually neglected) were included. On a long-term basis, the problem of true changes in genetic variance, especially in transfer plans, has not been solved.

Key words: Selection, genetic variance, inbreeding, nuclei, embryo transfer, dairy cattle.

\title{
I. Introduction
}

Depuis les 5 dernières années, la maîtrise des techniques de superovulation, de collecte et de transfert des æufs chez les bovins a considérablement progressé (HEYman et al., 1983 ; Chupin \& Procureur, 1983). Dans le même temps, les programmes de sélection chez les bovins laitiers ont eu tendance à s'intensifier et à se rapprocher du maximum de leurs possibilités techniques. Il est donc naturel de se demander si l'utilisation systématique des précédentes techniques ne doit pas être recommandée en complément des programmes habituels de sélection, en vue d'accroître le progrès génétique qu'ils induisent.

L'idée que suggère immédiatement l'existence de telles techniques est qu'il devient alors possible d'augmenter la pression de sélection le long des voies mère-fils et mère-fille de transmission des gènes. Un certain nombre d'auteurs se sont intéressés à ces aspects (LAND \& Hill, 1975 ; CUnNingham, 1976 ; Krausslich, 1976 ; Church \& Shea, 1977 ; Jandrain, 1977 ; Petersen \& Hansen, 1977 ; Mc Daniel \& Cassell, 1981 ; Van Vleck, 1981 ; MocQuot, 1982). Leurs conclusions sont en général négatives ou subordonnées à un abaissement très sensible du prix de revient du transfert (VAN VLECK, 1981). En effet, l'accroissement de la pression de sélection 
le long de la voie mère-fils n'entraîne qu'une augmentation faible $(5$ p. 100$)$ du progrès génétique global dans l'état actuel des techniques ( 3 à 5 veaux par an et par mère collectée) et dans le cas d'un programme de sélection déjà très efficace. Par ailleurs, les progrès potentiels apportés par un renforcement de la sélection le long de la voie mère-fille sont du même ordre, alors qu'ils supposent une utilisation généralisée de la technique, synonyme d'un faible coût pour qu'il puisse être supporté par les éleveurs, et de complexes échanges entre élevages. Une intensification considérable des techniques de superovulation (20 veaux/vache/an) entraînerait une amélioration sensible (de l'ordre de 15 p. 100) mais cette hypothèse n'est en général pas considérée comme réaliste par les auteurs précédents.

A l'opposé, Nicholas (1979), Nicholas \& Smith (1983) considèrent que l'utilisation du transfert d'œufs dans des noyaux fermés de sélection, concentrés dans un nombre limité d'élevages, permet de réaliser des progrès génétiques considérables, même en les comparant à ceux fournis par des programmes classiques performants. La supériorité pourrait être de l'ordre de 30 à 50 p. 100. Il s'agit en quelque sorte de la généralisation des résultats trouvés précédemment par LAND \& Hill (1975), ELSEN \& MocQuot (1976) sur bovins de race à viande, avec un programme de sélection de référence peu efficace, parce que ne faisant pas appel à l'insémination artificielle et au testage sur descendance.

Les résultats de Nicholas (1979), Nicholas \& Smith (1983) sont encourageants dans le sens où ils montrent que le transfert d'œufs pour être performant doit s'accompagner d'une réduction de l'intervalle de génération, handicap bien connu des programmes de sélection laitière c'est-à-dire concrètement d'une collecte et d'un transfert précoce des embryons. Tous leurs schémas reposent sur cette idée mais impliquent apparemment l'adhésion à une centralisation des programmes de sélection, en dehors des structures collectives de l'insémination artificielle et du contrôle laitier, telle que la propose HiNks (1978). Plus précisément, Nicholas (1979) envisageait dans sa première étude une variante où le testage des taureaux produits dans le noyau se fait sur descendance, à l'extérieur de celui-ci. Mais cette idée n'est pas reprise dans l'étude plus récente de Nicholas \& Smith (1983) où les taureaux du noyau sont sélectionnés sur ascendance d'après les performances des femelles du noyau.

Une telle organisation n'est sans doute pas indispensable dans le cas où les structures collectives mentionnées précédemment sont développées. Nous examinerons dans cet article si les idées fondamentales de Nicholas concernant le seul transfert d'embryons ne peuvent pas être purement et simplement transposées au cas général des programmes de sélection collectifs et décentralisés, revenant ainsi à une idée première de Nicholas. Notre critère essentiel de jugement sera le supplément de progrès génétique apporté par l'utilisation précoce du transfert d'embryons, en comparaison à un programme de type classique, délibérément poussé aux limites de ses possibilités.

Comme indiqué dans le titre, nous n'examinerons que les aspects techniques (niveaux génétiques, coefficients de consanguinité) liés à une utilisation systématique du transfert accéléré d'embryons dans les noyaux de sélection. Il s'agit là de la première étape indispensable sur laquelle il est nécessaire de s'appuyer si l'on veut répondre adéquatement aux questions posées par les sélectionneurs et qui concernent la rentabilité éventuelle au niveau de la population générale, de l'introduction systématique de techniques complexes et coûteuses dans les programmes de sélection. 


\section{Matériel et méthodes}

\section{A. Schéma de sélection de référence}

\section{Situation du schéma}

Le schéma décrit est supposé avoir atteint la phase asymptotique tant pour l'évolution des niveaux génétiques que pour celle des coefficients de consanguinité et de parenté.

\section{Description du schéma}

Il s'inspire du schéma décrit par Ducroce (1984) pour le cas d'une unité de sélection de taille moyenne en France. La sélection est alors supposée n'être effectuée que sur un seul caractère : la production laitière $\left(h^{2}=0,25\right)$. Cependant, il en diffère dans le sens d'une intensification encore plus poussée (tableaux 1, 2 et 3).

a) Intensités de sélection accrues le long des voies père-fils et père-fille de transmission des gènes

La cause essentielle de cette intensification repose sur un doublement supposé de la fraction active de la population. Pour 400000 vaches inséminées, on passe dans notre cas de 100000 à 200000 vaches inséminées et contrôlées. Dans ces conditions, l'unité de sélection teste 100 taureaux par an et non plus 50 .

\section{b) Réduction des intervalles de génération}

Le délai d'obtention des index laitiers passe de 6 à 5,5 ans : on considère que les inséminations de testage sont terminées à 18 mois et non à 2 ans. Par ailleurs, la durée d'utilisation des taureaux, une fois connu l'index, n'est que d'une année pendant laquelle tout le stock (50 000 doses/taureau) est épuisé.

\section{Evolution des niveaux génétiques}

L'utilisation de la formule de RENDEL \& RoBERTSON (1950), permet d'aboutir à un progrès génétique annuel de 0,226 écart-type génétique, soit une augmentation de 18 p. 100 par rapport au schéma maximaliste de Ducroce (tabl. 3). Les schémas classiques mentionnés par les auteurs cités précédemment se situent notablement en dessous du niveau envisagé ici. Seuls Van Vleck (1981), Nicholas \& SMith (1983) s'en rapprochent avec une valeur de 0,20 écart-type génétique pour le progrès génétique annuel.

Si le critère de sélection laitière est la quantité de matière utile (MU), telle que la définissent Poutous et al. (1981), [MU $=\frac{1}{2}$ (Quantité de matière grasse $+1,21$ Quantité de matière protéique)], l'écart-type génétique est d'environ $18 \mathrm{~kg}$ (BonAITI \& MoCQUOT, 1982) et la moyenne en première lactation d'une race comme la race Pie-Noir est de $173 \mathrm{~kg}$ (Anonyme, 1982). Le progrès génétique annuel représente 
TABLEAU 1

Paramètres généraux du schéma classique de référence.

General parameters of the conventional reference scheme.

\begin{tabular}{|c|c|c|}
\hline & de $\begin{array}{l}\text { Etude } \\
\text { Ducroce } \\
(1984)\end{array}$ & $\begin{array}{l}\text { Présente } \\
\text { étude }\end{array}$ \\
\hline \multicolumn{3}{|l|}{ Paramètres génétiques } \\
\hline Héritabilité de la production laitière $\ldots \ldots \ldots \ldots \ldots \ldots \ldots$ & \multicolumn{2}{|c|}{0,25} \\
\hline Répétabilité de la production laitière & \multicolumn{2}{|c|}{0,50} \\
\hline Corrélation génétique entre $1^{\text {re }}$ lactation et suivantes $\ldots \ldots$ & \multicolumn{2}{|c|}{1} \\
\hline \multicolumn{3}{|l|}{ Paramètres démographiques } \\
\hline Effectif de femelles inséminées $\ldots \ldots \ldots \ldots \ldots \ldots \ldots \ldots$ & \multicolumn{2}{|c|}{400000} \\
\hline Population active (femelles contrôlées et d'ascendance connue) & 100000 & 200000 \\
\hline Pourcentage de femelles en $1^{\text {re }}$ lactation issues de testage.. & \multicolumn{2}{|c|}{$10 \%$} \\
\hline Age au $1^{\text {er }}$ vêlage $\ldots \ldots \ldots \ldots \ldots \ldots \ldots \ldots \ldots \ldots \ldots \ldots \ldots \ldots \ldots \ldots \ldots \ldots \ldots$ & \multicolumn{2}{|c|}{2 ans } \\
\hline Intervalle entre vêlages $\ldots \ldots \ldots \ldots \ldots \ldots \ldots \ldots \ldots$ & \multicolumn{2}{|c|}{1 an } \\
\hline Nombre d'I.A. nécessaires pour obtenir un veau né vivant .. & \multicolumn{2}{|c|}{1,7} \\
\hline Mortalité des génisses de 0 à 2 ans $\ldots \ldots \ldots \ldots \ldots \ldots$ & \multicolumn{2}{|c|}{$15 \%$} \\
\hline Pyramide des âges des femelles (données du contrôle laitier) & $\mathrm{N}^{\circ}$ lactation & Pourcentage \\
\hline Sex-ratio & $\begin{array}{r}1 \\
2 \\
3 \\
4 \\
5 \\
6 \\
7 \\
8 \\
9 \\
10\end{array}$ & $\begin{array}{r}26 \\
21 \\
17 \\
13 \\
9 \\
6 \\
4 \\
2 \\
1 \\
1\end{array}$ \\
\hline$\cdots \cdots \cdots$ & \multicolumn{2}{|c|}{0,5} \\
\hline
\end{tabular}




\section{TABleau 2}

Paramètres détaillés du schéma classique de référence.

Detailed parameters of the conventional reference scheme.

\begin{tabular}{|c|c|c|}
\hline & $\begin{array}{l}\text { Etude } \\
\text { de Ducroce } \\
(1984)\end{array}$ & $\begin{array}{l}\text { Présente } \\
\text { étude }\end{array}$ \\
\hline \multicolumn{3}{|l|}{ Voir père-fils $\left(n^{\circ} 1\right)$} \\
\hline $\begin{array}{l}\text { Nombre de pères à taureau retenus par an } \ldots \ldots \ldots \ldots \ldots \\
\text { Durée moyenne d'utilisation des pères à taureau } \ldots \ldots \ldots\end{array}$ & \multicolumn{2}{|l|}{2 ans } \\
\hline Voir père-fille $\left(\begin{array}{ll}n^{\circ} & 2\end{array}\right)$ & & \\
\hline Nombre annuel de taureaux mis en testage sur descendance & 50 & 100 \\
\hline $\begin{array}{l}\text { Nombre moyen de filles en première lactation, par taureau } \\
\text { de testage } \ldots \ldots \ldots \ldots \ldots \ldots \ldots \ldots \ldots \ldots \ldots \ldots \ldots \ldots \ldots \ldots \ldots \ldots \ldots \ldots\end{array}$ & \multicolumn{2}{|c|}{52} \\
\hline $\begin{array}{l}\text { Elimination en station pour d'autres critères que ceux étudiés } \\
\text { (spermatogénèse, aspect sanitaire...) } \ldots \ldots \ldots \ldots \ldots \ldots \ldots\end{array}$ & \multicolumn{2}{|c|}{$25 \%$} \\
\hline Stock de semence disponible au moment de l'indexation . & \multicolumn{2}{|c|}{50000 doses } \\
\hline Age à l'indexation . & 6 ans & 5,5 ans \\
\hline Nombre maximal de doses utilisées/taureau et/an ... & 25000 & 50000 \\
\hline Durée maximale d'utilisation de la semence après indexation & 4 ans & 1 an \\
\hline Durée maximale de prélèvement des taureaux après indexation & 3 ans & 0 \\
\hline $\begin{array}{l}\text { Quantité moyenne prélevée par taureau et par an après } \\
\text { indexation } \ldots \ldots \ldots \ldots \ldots \ldots \ldots \ldots \ldots \ldots \ldots \ldots \ldots \ldots \ldots \ldots \ldots \ldots \ldots \ldots \ldots\end{array}$ & 15000 doses & 0 \\
\hline Besoin annuel en doses pour toute la population .... & \multicolumn{2}{|c|}{680000} \\
\hline $\begin{array}{l}\text { Nombre de taureaux de service } \ldots \\
\text { Voir mère-fils }\left(n^{\circ} 3\right)\end{array}$ & & 13 \\
\hline $\begin{array}{l}\text { Nombre de mères à taureau nécessaires par taurillon entrant } \\
\text { en station } \ldots \ldots \ldots \ldots \ldots \ldots \ldots \ldots \ldots \ldots \ldots \ldots \ldots \ldots \ldots \ldots \ldots \ldots \ldots \ldots \ldots \ldots\end{array}$ & \multicolumn{2}{|c|}{6} \\
\hline $\begin{array}{l}\text { Nombre de lactations connues nécessaires pour être mère à } \\
\text { taureau } \ldots \ldots \ldots \ldots \ldots \ldots \ldots \ldots \ldots \ldots \ldots \ldots \ldots \ldots \ldots \ldots \ldots \ldots \ldots \ldots \ldots \ldots \ldots \ldots \ldots \ldots \ldots\end{array}$ & \multicolumn{2}{|c|}{3} \\
\hline Voir mère-fille $\left(n^{\circ} 4\right)$ & & \\
\hline $\begin{array}{l}\text { Fraction des éliminations après la première et la seconde } \\
\text { lactation dues à un niveau laitier insuffisant } \ldots \ldots \ldots \ldots\end{array}$ & \multicolumn{2}{|c|}{$33 \%$} \\
\hline $\begin{array}{l}\text { Fraction des génisses connues sur ascendance } \\
\quad \text { - mère ayant au moins une lactation connue } \ldots \ldots \ldots \ldots \ldots \\
\text { - mère primipare } \ldots \ldots \ldots \ldots \ldots \ldots \ldots \ldots \ldots \ldots \ldots\end{array}$ & \multicolumn{2}{|c|}{$\begin{array}{l}75 \% \\
22 \%\end{array}$} \\
\hline
\end{tabular}


alors 4,08 kg MU, soit 2,4 p. 100 de la moyenne. D'après VAN VlecK (1981), le progrès génétique annuel maximum possible se situe dans la zone 2-2,5 p. 100 , et peut-être 3 p. 100 .

\section{TABleau 3}

Supériorités génétiques des parents sélectionnés (I) et intervalles de génération (L) pour le schéma classique de référence.

Genetic superiorities of the selected parents $(I)$ and generation intervals $(L)$ for the conventional reference scheme.

\begin{tabular}{|c|c|c|c|c|}
\hline \multirow{2}{*}{$\begin{array}{l}\text { Voie de transmission } \\
\text { des gènes }\end{array}$} & \multicolumn{2}{|c|}{$\begin{array}{l}\text { Etude de Ducroce } \\
(1984)\end{array}$} & \multicolumn{2}{|c|}{ Présente étude } \\
\hline & $\mathrm{L}$ & I & L & I \\
\hline 1 Père - fils .. & 7,5 & 1,74 & 6,75 & 1,99 \\
\hline 2 Père - fille . . & 7,5 & 1,17 & 6,30 & 1,43 \\
\hline 3 Mère - fils ... & 6,6 & 1,88 & 6,60 & 1,88 \\
\hline 4 Mère - fille .. & 5,6 & 0,43 & 5,60 & 0,43 \\
\hline \multirow{2}{*}{$\begin{array}{c}\text { Ensemble des voies } \ldots \ldots \ldots \ldots \ldots \ldots \ldots \ldots \\
\text { Progrès génétique annuel (en unité d'écart- } \\
\text { type génétique) } \ldots \ldots \ldots \ldots \ldots \ldots \ldots \ldots\end{array}$} & 6,8 & 1,305 & 6,31 & 1,432 \\
\hline & \multicolumn{2}{|c|}{0,192} & \multicolumn{2}{|c|}{0,226} \\
\hline
\end{tabular}

$\mathrm{L}=$ Intervalle de génération (année).

$\mathbf{I}=$ Supériorité génétique des parents (écart-type génétique).

4. Evolution des coefficients de consanguinité et de parenté

\section{a) Problèmes théoriques}

Le calcul strict de ces coefficients dans une population sélectionnée soulève de nombreuses difficultés. C'est pourquoi les algorithmes généralement proposés ne tiennent compte de la sélection qu'au travers de la réduction des effectifs d'animaux reproducteurs par rapport aux populations dont ils sont issus. Tout se passe donc comme si les coefficients considérés ne portaient que sur les loci correspondant à des gènes neutres vis-à-vis du caractère sélectionné, et en outre non liés aux gènes intéressés. Par ailleurs, le processus de sélection modifie la distribution des effectifs totaux en familles et introduit une variance supplémentaire de la taille des familles (Robertson, 1961 ; BuRRows, 1984). Ce dernier calcule cette variance pour le premier cycle de sélection seulement, les hypothèses de calcul n'étant plus vérifiées ultérieurement. 
b) Relations de récurrence asymptotiques

Comme il a été dit plus haut, on suppose que le schéma classique a atteint sa phase asymptotique. Si l'on désigne par $F_{t}$ le coefficient de consanguinité moyen des animaux nés l'année $t$, on a :

$$
\left(F_{t}-F_{t-1}\right) /\left(1-F_{t-1}\right)=1 / 2 N_{e} L
$$

où $\mathrm{N}_{\mathrm{e}}$ désigne l'effectif efficace par génération et où $\mathrm{L}$ désigne l'intervalle de génération (HILL, 1972).

HiLl (1979) a montré que l'effectif efficace par génération est donné par :

où :

$$
\begin{aligned}
1 / \mathrm{N}_{\mathrm{e}} & =(1 / 16 \mathrm{~m})\left[2+\sigma_{1}^{2}+2(\mathrm{~m} / \mathrm{f}) \operatorname{cov}(1,2)+(\mathrm{m} / \mathrm{f})^{2} \sigma_{2}^{2}\right] \\
& +(1 / 16 \mathrm{f})\left[2+(\mathrm{f} / \mathrm{m})^{2} \sigma_{3}^{2}+2(\mathrm{f} / \mathrm{m}) \operatorname{cov}(3,4)+\sigma_{4}^{2}\right]
\end{aligned}
$$

$\mathrm{m}$ est le nombre de mâles par génération,

f est le nombre de femelles par génération,

et où les $\sigma^{2}$ et Cov désignent les variances entre tailles de familles le long des 4 voies de transmission du progrès génétique,

voie $1=$ voie père-fils,

voie $2=$ voie père-fille,

voie $3=$ voie mère-fils,

voie $4=$ voie mère-fille.

La présence des taureaux de testage non retenus pour le service complique les calculs. D'une part, on ne peut retenir l'hypothèse classique de l'égalité des espérances de nombre de filles par taureau (calcul de $\sigma_{2}^{2}$ ) et d'autre part les effectifs de fils et de filles par taureau sont liés $(\operatorname{cov}(1,2) \neq 0)$.

Les calculs développés en annexe 1 permettent d'évaluer l'accroissement annuel du coefficient de consanguinité à 0,04 p. 100 .

\section{Principes généraux}

\section{B. Schémas de transfert d'œufs}

Ces schémas sont comme le schéma classique de référence consacrés à la sélection d'un caractère unique. La constitution du noyau de sélection où sont appliqués ces schémas a pour but la production de jeunes taureaux de testage, qui sont ensuite testés dans les troupeaux ordinaires du contrôle laitier, suivant les procédures habituelles exposées au paragraphe A. Dans ces conditions, il est tout à fait naturel de supposer que les modalités de la sélection le long de la voie père-fils ne sont pas affectées par l'utilisation du transfert d'embryons.

Par ailleurs, on suppose, à l'inverse de Nicholas \& SMITH (1983) que la localisation spatiale des femelles du noyau n'est pas limitée à quelques élevages, qu'elle peut être au contraire répartie sur un grand nombre d'élevages du contrôle laitier et qu'elle peut varier d'une génération à l'autre, notamment à l'occasion de la mișe en place des embryons. «Noyau de sélection» n'est donc pas synonyme de «Noyau d'éleveurs ». Cette supposition est effectuée pour préserver le caractère collectif des 
schémas de sélection français et pour se prémunir contre d'éventuels phénomènes d'interaction génotype-milieu, biaisant les performances des donneuses sélectionnées. Dans ces conditions, les femelles du noyau de sélection, y compris les donneuses, doivent avoir une carrière de productrice aussi peu affectée que possible par les opérations de sélection (dans le cas contraire, l'éleveur rejetterait le système ou demanderait des compensations financières coûteuses). En fin de compte, ce contexte général a un effet certain sur les modalités techniques des schémas que nous proposons. En particulier, nous ne proposons pas un nombre de récoltes excessif qui allongerait la période improductive ou l'intervalle entre vêlages : nous nous sommes limités à 2 récoltes.

Les modalités techniques des schémas ont été également directement influencées par d'autres types de préoccupation.

Nous avons jugé préférable de ne pas retenir comme donneuses sélectionnées les femelles ayant présenté certaines difficultés de reproduction, bien que les liaisons génétiques entre performances de reproduction sur génisses et sur multipares sont loin d'être très claires (JANSON, 1980; HANSEN et al., 1983). Les vaches non pleines au bout de 2 inséminations suivant les 2 collectes ne sont pas prises en considération, de même que l'ensemble de leurs descendants. Cette manière de procéder peut éventuellement contribuer à éviter d'introduire dans le programme certains gènes non favorables à la reproduction.

Par ailleurs, la possibilité d'une deuxième implantation d'embryons sur la même receveuse en cas d'échec de la première est admise de manière à diminuer le nombre total de receveuses nécessaires, ce qui contribue à diminuer le coût total de l'opération et surtout permet un meilleur choix des receveuses sur les critères d'âge et d'état général (HeYman et al., 1983) aux dépens toutefois d'un léger allongement de l'intervalle de génération.

\section{Schémas comparés}

\section{a) Schéma $A$ (exemple numérique en fig. 1)}

Toutes les femelles de renouvellement du noyau sont collectées à 16-18 mois et leurs embryons transférés immédiatement. Le choix des donneuses sélectionnées s'effectue à 6 mois de lactation. (A cette époque les produits issus de transfert ont 9 mois d'âge). Nous ferons l'approximation que cette sélection donne les mêmes résultats qu'une sélection sur lactation totale : en effet, les coefficients de corrélation génétique entre la production à 6 mois de lactation et la production totale sont au moins égaux à 0,95 (AURAN, 1976 ; DANEll, 1981). Le critère de choix des donneuses est uniquement la propre performance. Aucune performance des collatéraux n'est prise en considération pour ne pas risquer de sélectionner préférentiellement certaines familles, paternelles notamment et d'accélérer en conséquence l'augmentation des coefficients de consanguinité. Il s'agit là d'une précaution que nous avons choisi de prendre mais dans l'absolu, elle n'est nullement obligatoire.

La pression de sélection le long de la voie mère-fils et de la voie mère-fille est uniquement déterminée par les besoins de renouvellement, fixes pour la première voie (130 taureaux de 9 mois par an qui correspondent à 100 taureaux mis au testage après élimination de 25 p. 100 des taureaux sur la qualité de leur semence, 


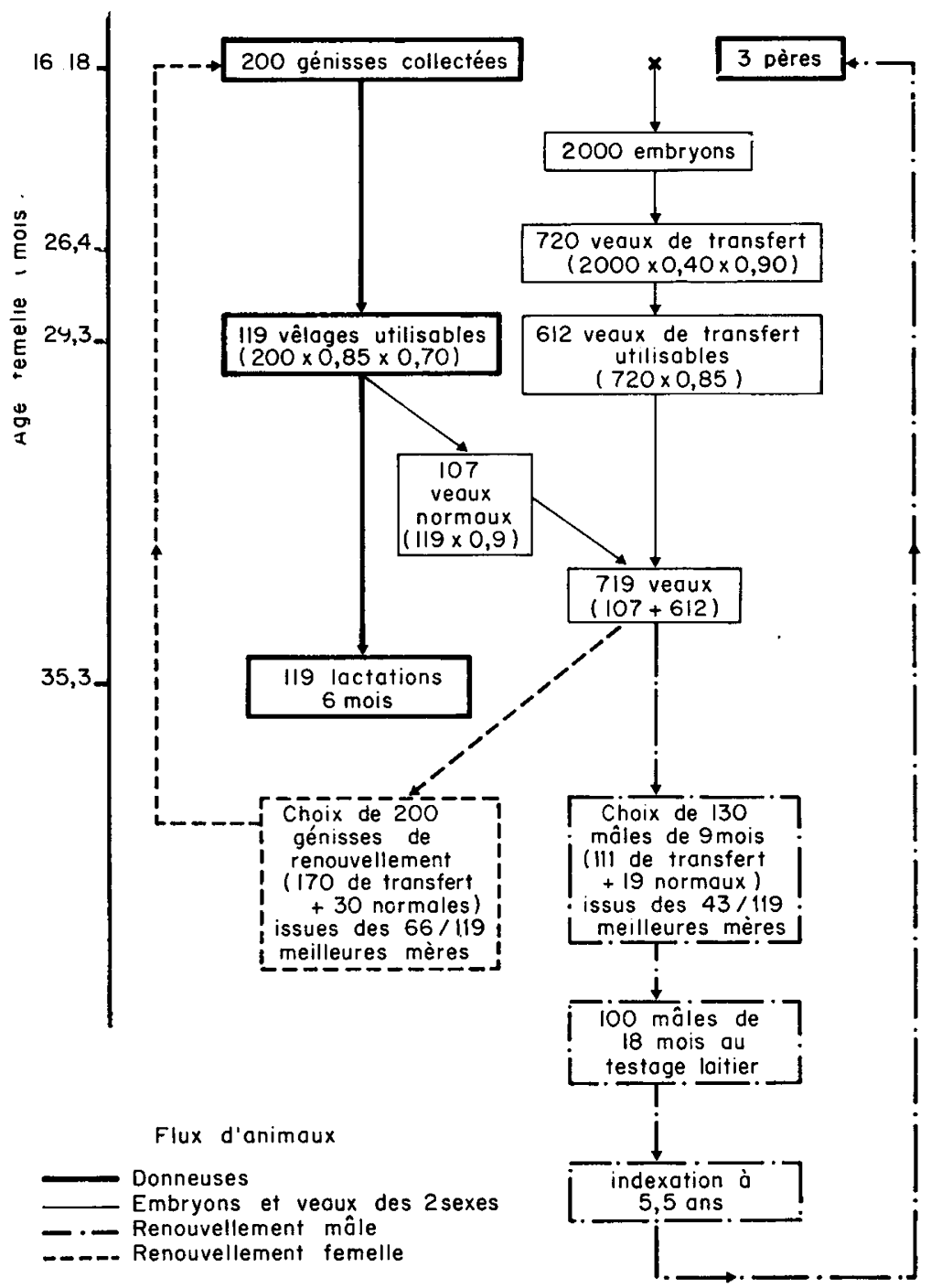

FIG. 1

Schéma de transfert A (les paramètres sont les suivants : taille du noyau $=200$; nombre d'embryons par récolte $=5$; taux de survie des embryons $=40 \mathrm{p} .100$ ).

Transfer scheme $A$ (with the following parameters : nucleus size $=200$; number of embryo per recovery $=5$; embryo survival rate $=40$ p. 100). 
suivant une procédure classiquement effectuée) et variables en fonction de la taille du noyau pour la deuxième voie.

Les veaux mâles et femelles de renouvellement sont à la fois des veaux normaux et des veaux issus de transfert, les pressions de sélection étant identiques pour ces 2 provenances. Il s'agit là d'une simplification qui affecte peu le progrès génétique, qui est proche de l'optimum, étant donné que les dates de naissance des veaux normaux et des veaux de transfert sont très proches (comme on le verra, ce n'est pas du tout le cas pour le schéma B). Ce schéma présente des analogies avec les schémas «juvéniles» de Nicholas \& SMith (1983).

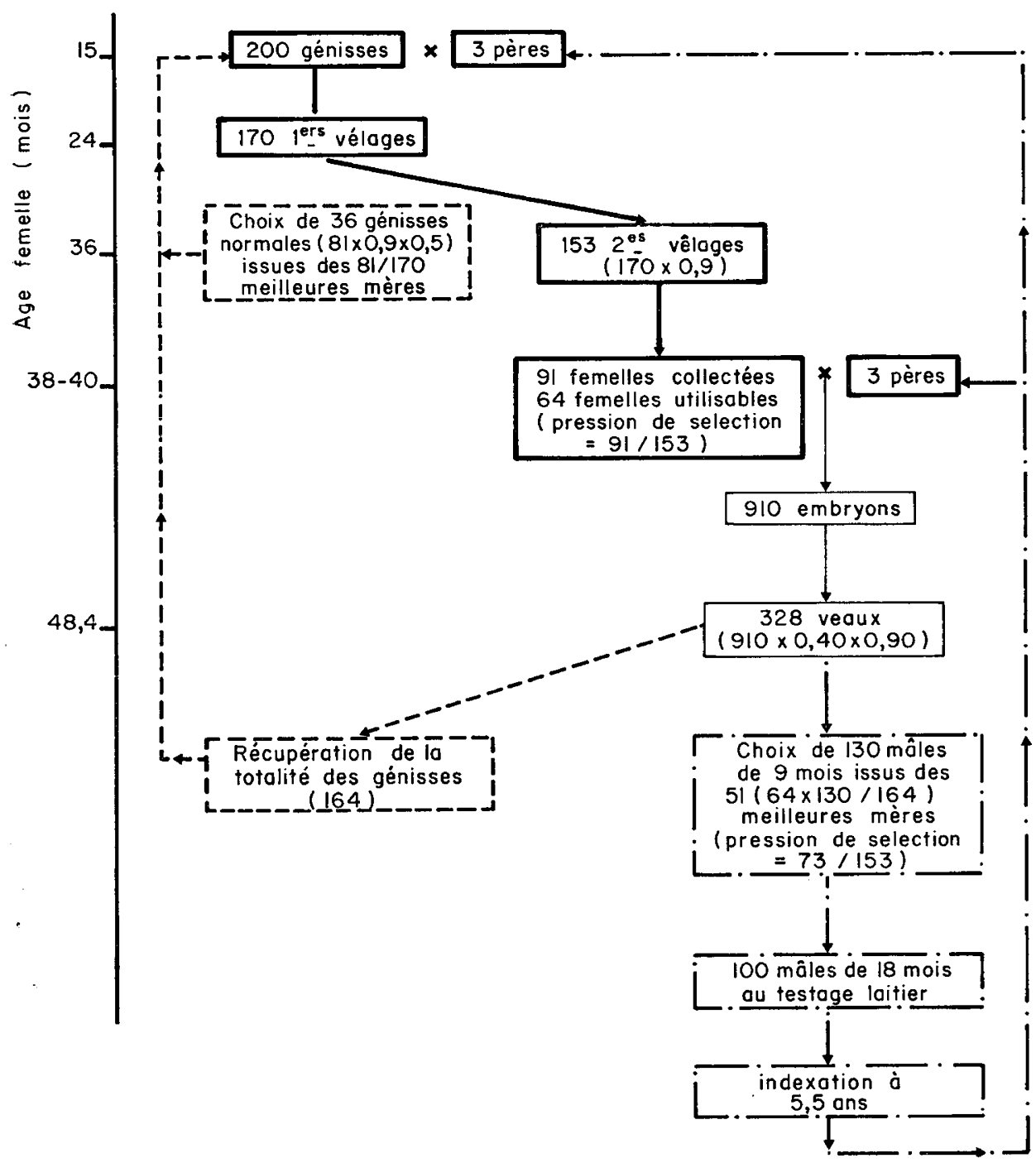

FIG. 2

Schéma de transfert $B$ (mêmes paramètres que pour $A$ ).

Transfer scheme $B$ (same parameters than for $A$ ). 


\section{b) Schéma $B$ (exemple numérique en fig. 2)}

Dans ce schéma, l'organisation technique est moins pesante et donc plus susceptible d'être acceptée. D'une part, on ne collecte que de manière à satisfaire exactement les besoins de renouvellement en mâles et en génisses. D'autre part, la destination future des veaux mâles est connue dès leur naissance (soit mise en testage, soit possibilité de vente en boucherie), ce qui limite les frais d'élevage.

D'une manière plus précise, les collectes ont lieu en début de deuxième lactation (au deuxième et au quatrième mois de lactation). Le choix s'effectue sur les performances en première lactation. Les veaux mâles de renouvellement sont tous issus de transfert, les veaux mâles normaux issus $\mathrm{du} 2^{\mathrm{e}}$ vêlage étant négligés. La pression de sélection sur les mères est déterminée de manière à fournir 130 taureaux. Les veaux femelles de renouvellement proviennent à la fois des veaux normaux et des veaux de transfert. A effectif constant de femelles de renouvellement, le progrès génétique annuel varie suivant le rapport : effectif de veaux femelles normaux / effectif de veaux femelles de transfert. Il existe un rapport optimal, qui est systématiquement considéré dans la suite des calculs. Il conduit à privilégier l'utilisation des veaux femelles normaux car ils diminuent l'intervalle de génération sur la voie mère-fille. Les algorithmes de calcul figurent en annexe 2.

Le schéma B rappelle les schémas «adultes» de Nicholas \& SMith. Nous n'avons pas retenu leur proposition d'effectuer les collectes au $10^{\mathrm{c}}$ mois de la $1^{\text {re }}$ lactation car l'intervalle entre premier et second vêlage aurait été très élevé (21 mois au minimum). Dans notre schéma, l'intervalle entre second et troisième vêlage est plus acceptable par l'éleveur (15 mois au minimum).

\section{c) Schéma $B^{\prime}$}

En partant d'une même taille de noyau, le schéma $B$ conduit à la mise en place d'un nombre plus réduit d'embryons que dans le schéma $A$. Dans le schéma $B^{\prime}$, au contraire, le nombre total d'embryons est le même que dans le schéma A alors que la taille du noyau est supérieure. Le calcul de la taille optimale est mentionné en annexe 2. C'est toujours ceté taille qui est prise en considération par la suite.

\section{d) Les schémas de Nicholas et Smith ou Moet} (Multiple Ovulation and Egg Transfer) comme les appellent leurs auteurs (exemple numérique en fig. 3)

A des fins de comparaison, nous avons simulé l'application de la série de MoET la plus efficace, notée JJ (Juvenile-Juvenile) par Nicholas \& SMITH. Le choix des femelles et des mâles de renouvellement est en effet très précoce et s'effectue uniquement sur ascendance, les performances correspondantes étant mesurées dans le cadre du noyau de sélection. Les veaux normaux sont par ailleurs négligés.

La transposition numérique précise du MOET JJ s'effectue dans les conditions suivantes :

- L'intervalle de génération mère-fils et mère-fille est égal à l'intervalle de génération correspondant pour les veaux de transfert nés dans le schéma $\mathrm{A}$.

— L'intervalle de génration père-fils et père-fille est égal à 1,75 an, l'âge moyen des taureaux à l'insémination étant supposé égal à 1 an. 


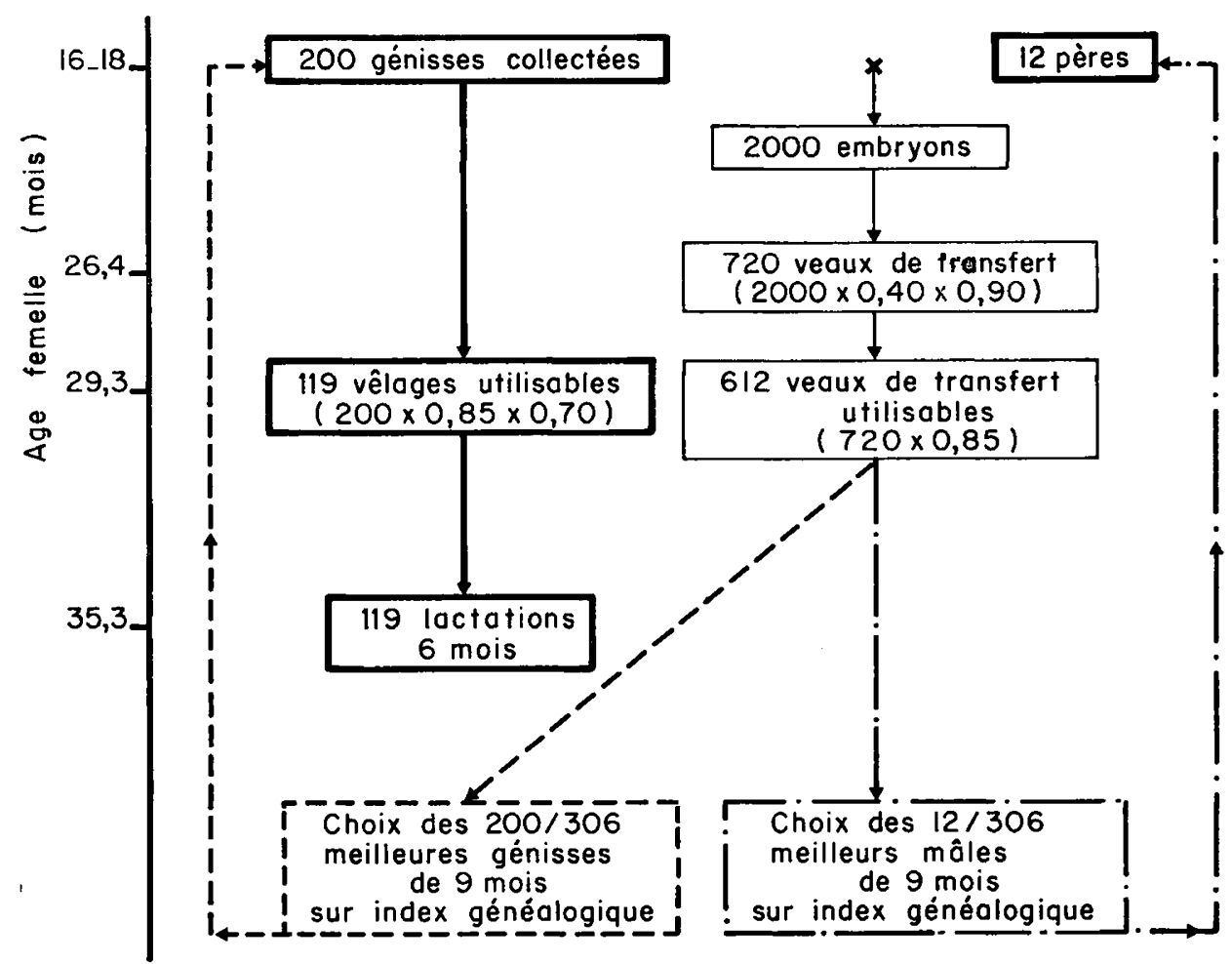

FIG. 3

Schéma de transfert MOET JJ16 (mêmes paramètres que pour A).

Transfer scheme MOET JJ16 (same parameters than for A).

- Aucun mâle n'est éliminé d'après l'examen morphologique de la semence.

- L'index sur ascendance est celui proposé par Nicholas \& SMITH : il tient compte des performances de la grand-mère maternelle ( 3 lactations), de la mère (1 lactation), des pleines sœurs de la mère (1 lactation), des demi-sœurs paternelíes de la mère (1 lactation). Cette méthode est plus efficace qu'une sélection à 2 étapes, d'abord sur le niveau laitier des grands-mères maternelles puis sur celui des mères et de leurs collatérales.

- Les paramètres de variabilité de la production laitière, les paramètres de viabilité et de reproduction sont les mêmes que dans les autres schémas.

Par ailleurs, il est nécessaire de se fixer le nombre de taureaux utilisés. Au vu des résultats de Nicholas \& SMith, nous avons retenu les variantes 16 et 32 pour le rapport nombre de femelles/nombre de taureaux, car les plus performantes.

Finalement, aux schémas précédents (A, B, B') nous adjoignons 2 MoET notés JJ 16 et JJ 32. 


\section{Paramètres envisagés}

a) Le nombre d'embryons transférables obtenu par vache traitée

La valeur de référence est considérée comme correspondant à 5 oufs transférables/génisse traitée/collecte (ce qui correspond à 10 œufs par génisse traitée puisque nous supposons qu'il y a toujours 2 récoltes). Elle est effectivement déjà obtenue en France après un traitement hormonal à la FSH (CHUPIN \& Procureur, 1983). Une hypothèse pessimiste correspond à 4 œufs et une hypothèse très optimiste à 8 œufs. Cette valeur pourra peut-être être atteinte dans le futur. Dans l'état actuel des techniques, elle ne pourrait être obtenue qu'en utilisant la technique de duplication des œufs, avec obtention éventuelle de couples de vrais jumeaux, ce qui poserait à grande échelle des problèmes génétiques nouveaux ou amènerait à revoir les calculs (cas de l'élimination d'un des jumeaux).

\section{b) Le taux de survie des embryons}

Il varie avec de multiples facteurs, notamment la technique de transfert (cervicale/chirurgicale), l'utilisation ou non de la congélation, l'habileté des équipes techniques tant au niveau du transfert que de la congélation (HEYMAN et al., 1983). Toutes causes de variation confondues, la gamme de variation la plus communément rencontrée se situe entre 40 p. 100 et 60 p. 100 de survie.

\section{c) La taille du noyau}

Nous entendons par là l'effectif de femelles de renouvellement. Pour fixer les idées, il correspond au nombre de génisses sélectionnées de 1 an et ceci quelque soit le schéma du transfert envisagé. Certaines tailles de noyau sont insuffisantes pour assurer la fourniture de veaux mâles pour le testage. Le paramètrage ne peut donc pas être totalement a priori. Compte tenu des besoins en veaux mâles pour notre cas particulier et des paramètres de démographie utilisés, nous avons donné à la taille du noyau trois valeurs : 100,200 et 400 .

d) Le nombre total d'embryons transférés

Trois valeurs seront considérées : 1000,1500 et 2000 embryons.

\section{Constantes}

- Sex-ratio . . ............... 50 p. 100

- Mortalité des veaux .............. 10 p. 100

- Proportion de génisses inséminées vêlant après 1 ou 2 inséminations $\ldots \ldots \ldots \ldots \ldots \ldots .65$ p. 100

- Taux de réforme entre le premier et le second vêlage $\ldots \ldots \ldots \ldots \ldots \ldots \ldots \ldots \ldots \ldots$ p. 100

- Proportion de donneuses ne donnant aucun embryon transférable .............. 30 p. 100 (Heyman et al., 1983)

- Intervalle entre premier et second vêlage .. 1 an 
- Taux d'élimination des taureaux en station pour problèmes de reproduction ..........

- Coefficient d'héritabilité de la production lai-

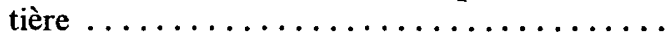

- Coefficient de répétabilité $\ldots \ldots \ldots \ldots \ldots \quad 0,50$

25 p. 100

0,25 (Revue de Maijala \& HanNA, 1974)

5. Calcul du progrès génétique asymptotique (en unité d'écart-type génétique)

a) Schémas $A, B, B^{\prime}$

On applique la formule de Rendel \& Robertson (1950) :

$$
\Delta \mathrm{G}=\left(\mathrm{I}_{1}+\mathrm{I}_{2}+\mathrm{I}_{3}+\mathrm{I}_{4}\right) / \mathrm{L}_{1}+\mathrm{L}_{2}+\mathrm{L}_{3}+\mathrm{L}_{4}
$$

où les $I$ désignent les supériorités génétiques des parents (exprimées en unité d'écarttype génétique) et où les $\mathrm{L}$ désignent les intervalles de génération correspondant le long des 4 voies père-fils (1), père-fille (2), mère-fils (3), mère-fille (4).

On a toujours $I_{1}=I_{2}=1,99$ et $L_{1}=L_{2}=6,8$ (cf. voie père-fils du schéma classique de référence).

Dans le schéma A $\mathrm{L}_{3}=\mathrm{L}_{4}$.

Dans les schémas $B$ et $B^{\prime}$, cette égalité n'est pas vérifiée (cf. annexe 2).

b) Schémas $J J$

On a dans ce cas :

$$
\begin{aligned}
& \mathrm{I}_{1}=\mathrm{I}_{2}=\text { notablement inférieur à } 1,99 \\
& \mathrm{~L}_{1}=\mathrm{L}_{2}=1,75 \\
& \mathrm{~L}_{3}=\mathrm{L}_{4}=\mathrm{L}_{41} \text { (cf. annexe } 2 \text { ) }
\end{aligned}
$$

6. Calcul de l'augmentation annuelle asymptotique du coefficient de consanguinité

Une mesure pratique simple qui pourrait être appliquée pour limiter la progression de ces coefficients consiste à utiliser systématiquement un taureau différent à chacune des 2 collectes. Cette contrainte complique un peu l'établissement des formules de calcul, dont le détail est donné en annexe 3.

Si l'on désigne par $m_{a}$ et $f_{a}$ les effectifs annuels de mâles et de femelles sélectionnés, par $\mathrm{L}$ l'intervalle de génération, l'évolution asymptotique annuelle du coefficient de consanguinité $\mathrm{F}$ est égale approximativement à :

$$
\Delta f \simeq\left(3 m_{a}+2 f_{a}\right) / 16 L^{2} m_{a} f_{a}
$$

7. Calcul des niveaux génétiques annuels dans le noyau

Ce calcul est effectué en utilisant la méthodologie de Hill (1974) et de ElseN \& MocQuot (1974), qui s'applique à des groupes d'animaux ou cohortes. 


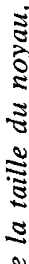

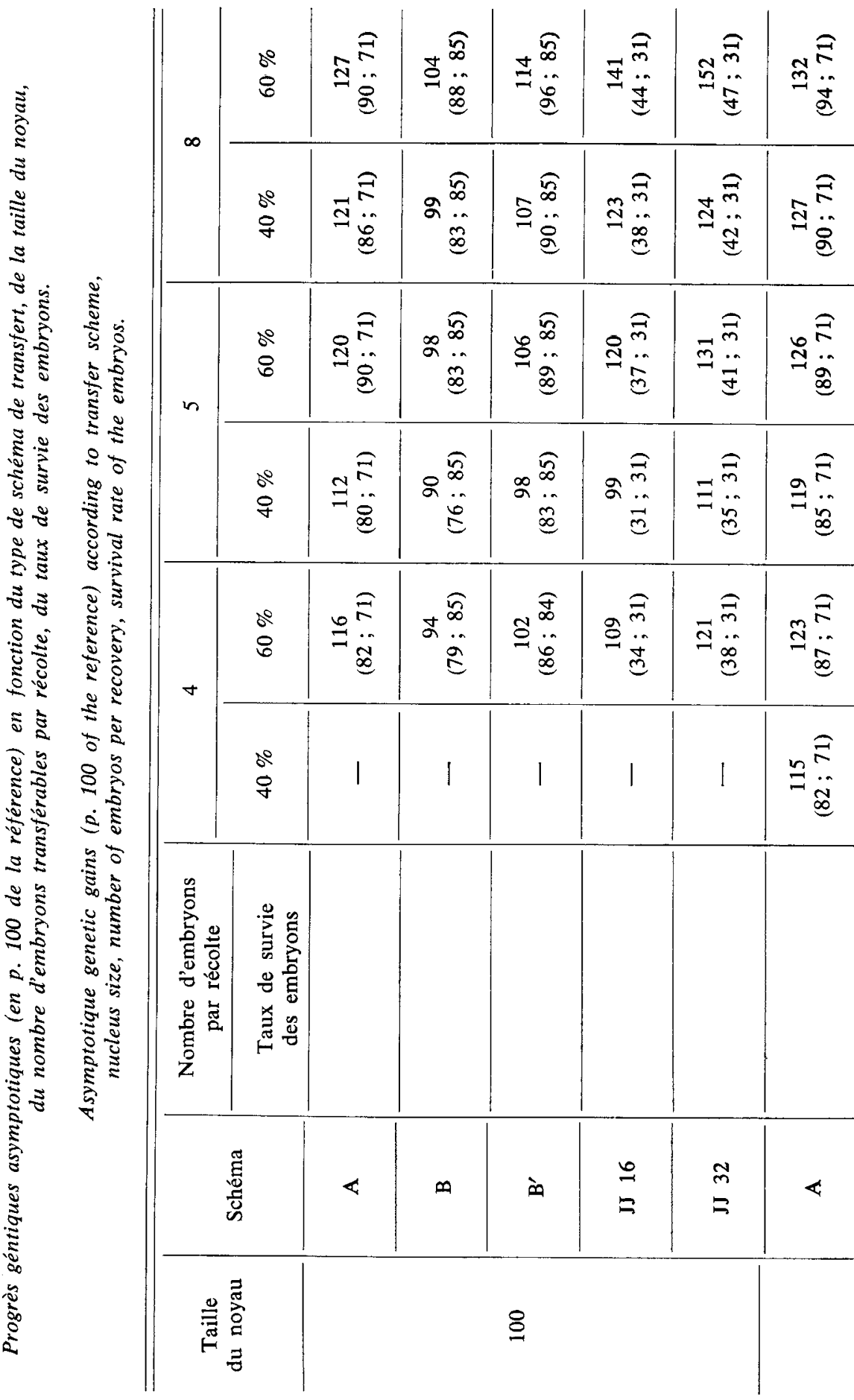


Dans notre cas, les intervalles de générations sont courts et même très courts dans certains cas. C'est pourquoi, la cohorte élémentaire prise en considération correspond à une durée très courte : 1 mois. Par ailleurs, des interpolations linéaires ont été effectuées sur les fréquences de ces cohortes de telle façon que les intervalles de génération correspondant à ce processus soient rigoureusement égaux aux intervalles de génération réels, pris en considération dans le calcul du progrès génétique asymptotique. Des jeux d'essais effectués sur des périodes de 100 ans ont montré une excellente concordance entre les progrès génétiques asymptotiques évalués par les 2 méthodes.

Dans ces conditions, la vitesse d'obtention du régime asymptotique a été évaluée en calculant les coefficients de régression du niveau génétique sur l'année pour 3 périodes adjacentes (années 0 à 10, 10 à 20, 20 à 30) et en les comparant au progrès génétique annuel obtenu par la formule de RENDEL \& RoBERTSON.

\section{Résultats}

\section{A. Progrès génétiques annuels asymptotiques}

\section{Sans contrainte}

Les progrès génétiques obtenus, exprimés en p. $100 \mathrm{du}$ progrès génétique fourni par le schéma classique de référence, figurent au tableau 4. En dessous de ces valeurs, figurent entre parenthèses les supériorités génétiques moyennes et les intervalles de génération moyens le long des 4 voies, exprimés en p. 100 des valeurs homologues obtenues dans le cas du schéma classique. A titre d'exemple numérique, des résultats plus détaillés concernant la combinaison $(200,5,40$ p. 100) sont montrés dans le tableau 5.

Dans la plupart des cas, les schémas envisagés sont d'une efficacité supérieure au schéma classique. Les supériorités génétiques le long des 4 voies sont inférieures ou même très inférieures à leurs homologues du schéma classique, cependant la réduction de l'intervalle de génération est dans la plupart des cas encore plus importante, ce qui fait que le résultat net des 2 phénomènes est celui mentionné ci-dessus. L'intérêt principal de ces types de schéma est donc bien la réduction de l'intervalle de génération.

La comparaison des schémas $\mathrm{A}, \mathrm{B}$ et $\mathrm{B}^{\prime}$ à l'intérieur d'une combinaison donnée taille du noyau $\times$ nombre d'embryons $\times$ taux de survie des embryons permet de mettre toujours en évidence la nette supériorité du schéma A sur le schéma B. Alors que le premier est toujours supérieur au schéma classique (d'au moins 10 p. 100), le second peut lui être inférieur, et même notablement (-10 p. 100) dans certains cas. La pratique de l'organisation du schéma B sur une taille de noyau plus importante, avec un nombre total d'embryons transférés identique au schéma A (schéma $B^{\prime}$ ) amćliore un peu la situation mais sans pouvoir rattraper le niveau du schéma A. L'inefficacité relative des schémas $\mathrm{B}$ et $\mathrm{B}^{\prime}$ tient en effet à une réduction insuffisante de l'intervalle de génération $(-15$ p. 100) comparée à celle fournie par le schéma A (-29 p. 100). 


\section{TABleaU 5}

Détail des modalités de calcul du progrès génétique asymptotique pour un exemple (taille du noyau $=200$; nombre d'embryons par récolte $=5$; taux de survie des embryons $=40$ p. 100).

Numerical example of calculating asymptotic genetic gain (nucleus size $=200$; number of embryos per recovery $=5$; survical rate of the embryos $=40 \mathrm{p} .100$ ).

\begin{tabular}{|c|c|c|c|c|c|}
\hline & \multicolumn{5}{|c|}{ Schéma } \\
\hline & A & B & $\mathbf{B}^{\prime}$ & JJ 16 & JJ 32 \\
\hline $\begin{array}{c}\text { Nombre de mâles de renouvelle- } \\
\text { ment } \ldots \ldots \ldots \ldots \ldots \ldots \ldots \ldots \\
- \text { normaux } \ldots \ldots \ldots \ldots \ldots \ldots \\
- \text { de transfert } \ldots \ldots \ldots \ldots \ldots \\
\end{array}$ & $\begin{array}{r}130 \\
19,4 \\
110,6 \\
\end{array}$ & $\begin{array}{r}130 \\
0 \\
130 \\
\end{array}$ & $\begin{array}{r}130 \\
0 \\
130 \\
\end{array}$ & $\begin{array}{c}12,5 \\
0 \\
12,5\end{array}$ & $\begin{array}{l}6,25 \\
0 \\
6,25\end{array}$ \\
\hline $\begin{array}{l}\text { Nombre de femelles de renouvelle- } \\
\text { ment } \ldots \ldots \ldots \ldots \ldots \ldots \ldots \ldots \ldots \\
- \text { normales } \ldots \ldots \ldots \ldots \ldots \ldots \\
- \text { de transfert } \ldots \ldots \ldots \ldots \ldots \\
\end{array}$ & $\begin{array}{r}200 \\
29,8 \\
170,2\end{array}$ & $\begin{array}{r}200 \\
36,4 \\
163,6 \\
\end{array}$ & $\begin{array}{l}461,7 \\
101,7 \\
360\end{array}$ & $\begin{array}{r}200 \\
0 \\
200\end{array}$ & $\begin{array}{r}200 \\
0 \\
200\end{array}$ \\
\hline Nombre de pères des mâles ...... & 3 & 3 & 3 & 12,5 & 6,25 \\
\hline Nombre de mères utilisables $\ldots$. & 119 & 107,1 & 247,2 & 119 & 119 \\
\hline $\begin{array}{l}\text { Nombre de mères de mâles nor- } \\
\operatorname{maux} \ldots \ldots \ldots \ldots \ldots \ldots \ldots \ldots \ldots \ldots \ldots\end{array}$ & 43 & 一 & - & 一 & 一 \\
\hline $\begin{array}{c}\text { Nombres de mères de mâles de } \\
\text { transfert } \ldots \ldots \ldots \ldots \ldots \ldots \ldots \ldots\end{array}$ & 43 & 50,6 & 50,6 & 4,9 & 2,4 \\
\hline $\begin{array}{c}\text { Nombre de mères de femelles nor- } \\
\text { males } \ldots \ldots \ldots \ldots \ldots \ldots \ldots \ldots \ldots \ldots\end{array}$ & 66,2 & 80,9 & 226 & - & - \\
\hline $\begin{array}{c}\text { Nombre de mères des femelles de } \\
\text { transfert } \ldots \ldots \ldots \ldots \ldots \ldots \ldots \ldots \\
\end{array}$ & 66,2 & 63,6 & 140 & 66,2 & 66,2 \\
\hline $\begin{array}{l}\text { Supériorités génétiques } \\
\text { (en unité d'écart-type génétique) } \\
\text { - voie père-fils ou père-fille } .\end{array}$ & & & & & \\
\hline $\begin{array}{l}\text { — voie père-fils ou pere-fille } \ldots \\
\text { — voie mère-fils } \ldots \ldots \ldots \ldots\end{array}$ & $\begin{array}{l}1,990 \\
0,518\end{array}$ & $\begin{array}{l}1,990 \\
0,422\end{array}$ & $\begin{array}{l}1,990 \\
0,694\end{array}$ & $\begin{array}{l}0,701 \\
0,185\end{array}$ & $\begin{array}{l}0,804 \\
0,188\end{array}$ \\
\hline - voie mère-fille normale .... & 0,355 & 0,208 & 0,208 & - & - \\
\hline — voie mère-fille de transfert ... & 0,355 & 0,422 & 0,326 & 0,185 & 0,188 \\
\hline $\begin{array}{l}\text { Intervalles de génération } \\
\quad \text { (en année) : }\end{array}$ & & & & & \\
\hline $\begin{array}{l}\text { — voie père-fils ou père-fille } \ldots \\
\text { — voie mère-fils } \ldots \ldots \ldots \ldots \ldots\end{array}$ & $\begin{array}{l}6,75 \\
2,235\end{array}$ & $\begin{array}{l}6,75 \\
4,031\end{array}$ & $\begin{array}{l}6,75 \\
4,031\end{array}$ & $\begin{array}{l}1,75 \\
2,198\end{array}$ & $\begin{array}{l}1,75 \\
2,198\end{array}$ \\
\hline — voie mère-fille normale .... & 2,444 & 3 & 3 & - & - \\
\hline _ voie mère-fille de transfert . . & 2,198 & 4,031 & 4,031 & 2,198 & 2,198 \\
\hline
\end{tabular}




\section{TABLEAU 6}

Progrès génétique en p. 100 de la référence ( $1^{\text {ro }}$ ligne) et taille du noyau ( $2^{e}$ ligne) en fonction du nombre total d'embryons transférés, du type de schéma de transfert, du nombre d'embryons par récolte, du taux de survie des embryons.

Asymptotic genetic gains (first line) and nucleus size (second line) according to total number of transferred embryos, transfer scheme, nucleus size, number of embryos per recovery, survival rate of the embryos.

\begin{tabular}{|c|c|c|c|c|c|c|c|c|}
\hline \multirow{2}{*}{$\begin{array}{l}\text { Nombre } \\
\text { d'embryons } \\
\text { transférés }\end{array}$} & \multirow[b]{2}{*}{ Schéma } & $\begin{array}{c}\text { Nombre d'embryons } \\
\text { par récolte }\end{array}$ & \multicolumn{2}{|c|}{4} & \multicolumn{2}{|c|}{5} & \multicolumn{2}{|c|}{8} \\
\hline & & $\begin{array}{l}\text { Taux de survie } \\
\text { des embryons }\end{array}$ & $40 \%$ & $60 \%$ & $40 \%$ & $60 \%$ & $40 \%$ & $60 \%$ \\
\hline \multirow{5}{*}{1000} & A & & $\begin{array}{l}111 \\
125\end{array}$ & $\begin{array}{l}119 \\
125\end{array}$ & $\begin{array}{l}112 \\
100\end{array}$ & $\begin{array}{l}120 \\
100\end{array}$ & $\begin{array}{r}116 \\
63\end{array}$ & $\begin{array}{r}123 \\
63\end{array}$ \\
\hline & B & & $\begin{array}{r}88 \\
125\end{array}$ & $\begin{array}{r}96 \\
125\end{array}$ & $\begin{array}{r}90 \\
100\end{array}$ & $\begin{array}{r}98 \\
100\end{array}$ & $\begin{array}{l}93 \\
63\end{array}$ & $\begin{array}{r}100 \\
63\end{array}$ \\
\hline & $\mathbf{B}^{\prime}$ & & $\begin{array}{r}95 \\
235\end{array}$ & $\begin{array}{l}104 \\
342\end{array}$ & $\begin{array}{r}98 \\
232\end{array}$ & $\begin{array}{l}106 \\
335\end{array}$ & $\begin{array}{l}104 \\
226\end{array}$ & $\begin{array}{l}111 \\
324\end{array}$ \\
\hline & JJ 16 & & $\begin{array}{r}86 \\
125\end{array}$ & $\begin{array}{l}109 \\
125\end{array}$ & $\begin{array}{r}99 \\
100\end{array}$ & $\begin{array}{l}120 \\
100\end{array}$ & $\begin{array}{r}123 \\
63\end{array}$ & $\begin{array}{r}141 \\
63\end{array}$ \\
\hline & JJ 32 & & $\begin{array}{r}98 \\
125\end{array}$ & $\begin{array}{l}121 \\
125\end{array}$ & $\begin{array}{l}111 \\
100\end{array}$ & $\begin{array}{l}131 \\
100\end{array}$ & $\begin{array}{r}134 \\
63\end{array}$ & $\begin{array}{r}152 \\
63\end{array}$ \\
\hline \multirow{5}{*}{1500} & A & & $\begin{array}{l}115 \\
188\end{array}$ & $\begin{array}{l}122 \\
188\end{array}$ & $\begin{array}{l}117 \\
150\end{array}$ & $\begin{array}{l}123 \\
150\end{array}$ & $\begin{array}{r}120 \\
95\end{array}$ & $\begin{array}{r}127 \\
95\end{array}$ \\
\hline & B & & $\begin{array}{r}93 \\
188\end{array}$ & $\begin{array}{l}100 \\
188\end{array}$ & $\begin{array}{r}95 \\
150\end{array}$ & $\begin{array}{l}101 \\
150\end{array}$ & $\begin{array}{l}98 \\
95\end{array}$ & $\begin{array}{r}104 \\
95\end{array}$ \\
\hline & $B^{\prime}$ & & $\begin{array}{r}98 \\
352\end{array}$ & $\begin{array}{l}106 \\
511\end{array}$ & $\begin{array}{l}101 \\
347\end{array}$ & $\begin{array}{l}108 \\
502\end{array}$ & $\begin{array}{l}107 \\
337\end{array}$ & $\begin{array}{l}113 \\
485\end{array}$ \\
\hline & JJ 16 & & $\begin{array}{r}86 \\
188\end{array}$ & $\begin{array}{l}109 \\
188\end{array}$ & $\begin{array}{r}99 \\
150\end{array}$ & $\begin{array}{l}120 \\
150\end{array}$ & $\begin{array}{r}123 \\
95\end{array}$ & $\begin{array}{r}141 \\
95\end{array}$ \\
\hline & JJ 32 & & $\begin{array}{r}98 \\
188\end{array}$ & $\begin{array}{l}121 \\
188\end{array}$ & $\begin{array}{l}111 \\
150\end{array}$ & $\begin{array}{l}131 \\
150\end{array}$ & $\begin{array}{r}134 \\
95\end{array}$ & $\begin{array}{r}152 \\
95\end{array}$ \\
\hline \multirow{5}{*}{2000} & A & & $\begin{array}{l}118 \\
250\end{array}$ & $\begin{array}{l}124 \\
250\end{array}$ & $\begin{array}{l}119 \\
200\end{array}$ & $\begin{array}{l}126 \\
200\end{array}$ & $\begin{array}{l}123 \\
126\end{array}$ & $\begin{array}{l}129 \\
126\end{array}$ \\
\hline & B & & $\begin{array}{r}96 \\
250\end{array}$ & $\begin{array}{l}102 \\
250\end{array}$ & $\begin{array}{r}97 \\
200\end{array}$ & $\begin{array}{l}103 \\
200\end{array}$ & $\begin{array}{l}101 \\
126\end{array}$ & $\begin{array}{l}106 \\
126\end{array}$ \\
\hline & $\mathrm{B}^{\prime}$ & & $\begin{array}{l}100 \\
468\end{array}$ & $\begin{array}{l}108 \\
680\end{array}$ & $\begin{array}{l}103 \\
462\end{array}$ & $\begin{array}{l}110 \\
667\end{array}$ & $\begin{array}{l}108 \\
448\end{array}$ & $\begin{array}{l}115 \\
645\end{array}$ \\
\hline & JJ 16 & & $\begin{array}{r}86 \\
250\end{array}$ & $\begin{array}{l}109 \\
250\end{array}$ & $\begin{array}{r}99 \\
200\end{array}$ & $\begin{array}{l}120 \\
200\end{array}$ & $\begin{array}{l}123 \\
126\end{array}$ & $\begin{array}{l}141 \\
126\end{array}$ \\
\hline & JJ 32 & & $\begin{array}{r}98 \\
250\end{array}$ & $\begin{array}{l}121 \\
250\end{array}$ & $\begin{array}{l}111 \\
200\end{array}$ & $\begin{array}{l}121 \\
200\end{array}$ & $\begin{array}{l}134 \\
126\end{array}$ & $\begin{array}{l}152 \\
126\end{array}$ \\
\hline
\end{tabular}


La taille du noyau est un facteur essentiel de variation. Si les performances de reproduction sont mauvaises, elle peut être tout à fait insuffisante à pourvoir aux besoins en taureaux : c'est le cas d'une des combinaisons envisagées (100 génisses, 4 embryons par récolte, 40 p. 100 de survie). Dans la situation de référence (5 embryons par récolte), la taille a un effet très net, quoique suivant la règle des rendements décroissants $(+5-6$ points entre 100 et $200,+4$ points entre 200 et 400$)$. La cause première en est l'intensification de la sélection le long de la voie mèrefils, puisque les besoins en taureaux sont fixés. A taille et taux de réussite fixés, le nombre d'embryons a un effet sensible et curvilinéraire ( +4 points entre 4 et 5 embryons et +6 points entre 5 et 8 embryons). A taille et nombre d'embryons fixés, les variations entre les extrêmes supposés de taux de réussite ont un effet notable (amplitude de 6 à 8 points suivant la taille). Dans la zone de la situation de référence (5 embryons par collecte), une augmentation d'un point du taux de réussite a le même effet que celle de 0,1 à 0,2 embryon supplémentaire par récolte.

Les MoET simulés à des fins de comparaison (JJ 16 et $\mathrm{JJ} 32$ ) sont généralement supérieurs au schéma classique, ce qui confirme les résultats de Nicholas \& Smith, en dépit d'une part que notre schéma classique est encore plus intensif que le leur et d'autre part que nous envisageons des hypothèses techniques en moyenne plus pessimistes : dans notre situation de référence, le nombre de veaux utilisables par vache est de 4,5 alors qu'il est de 8 pour ces auteurs. Les MOET montrent une grande sensibilité (coefficient de régression du progrès génétique par rapport au paramètre) aux résultats techniques en matière de transfert : ils sont approximativement 3 fois plus sensibles au nombre d'embryons et 2 fois plus sensibles au taux de survie que les schémas de transfert A, B, B'. Ceci est parfaitement normal car les performances de reproduction affectent alors les 4 voies de transmission du progrès génétique, par rapport à 2 seulement pour ces 3 schémas. Dans la situation de référence, le schéma JJ 32 est pratiquement équivalent au schéma A. Le schéma JJ 16 est bien entendu inférieur au schéma JJ 32 puisque la pression de sélection des mâles sur ascendance est 2 fois plus faible.

\section{A nombre total d'embryons constant}

Il est possible que dans l'ensemble des coûts afférents à la mise en place de tels schémas, ce soit le poste «mise en place des embryons» qui soit le plus important. Dans cette éventualité, on peut effectuer les comparaisons à nombre total d'embryons constant.

Le tableau 6 montre que le classement des schémas n'est pas modifié. Comme on pouvait s'y attendre, la mise en œuvre de cette contrainte fait baisser l'intérêt marginal du nombre d'embryons par récolte comparativement à celui du taux de réussite : une augmentation d'un point de taux de réussite équivaut alors à celle de 0,4 embryon supplémentaire. La mise en place du schéma A devrait s'effectuer à partir d'un nombre assez important d'embryons (minimum de l'ordre de 1500 ) pour que l'amélioration par rapport au schéma classique soit sensible (15 p. 100 et plus), même en cas de performances techniques de reproduction relativement défavorables.

\section{B. Augmentation annuelle asymptotique du coefficient de consanguinité}

Les résultats du tableau 7 montrent que d'une manière générale l'augmentation du coefficient de consanguinité est plus forte que pour le schéma classique $(0,04$ 
p. 100 par an). Les schémas $A, B$ et $B^{\prime}$ présentent des valeurs qui ne dépendent pratiquement ni de la taille du noyau ni des paramètres de reproduction. En fait, ce sont essentiellement le nombre de pères et l'intervalle de génération qui interviennent. En effet, le nombre de femelles est suffisamment grand pour que $3 \mathrm{~m}_{\mathrm{a}}=9$ soit négligeable devant $2 f_{a}$ et :

$$
\Delta \mathrm{f} \simeq 2 / 16 \mathrm{~L}^{2} \mathrm{~m}_{\mathrm{a}}=1 / 24 \mathrm{~L}^{2}
$$

L'augmentation du coefficient de consanguinité résultant des schémas JJ 16 et JJ 32 est élevée, et même très élevée dans certains cas. Ceci provient du fait que l'intervalle de génération est très court ( 2 fois moins élevé que celui du schéma $A$, 3 fois moins élevé que celui du schéma classique) et du fait que les taureaux pères sont tirés dans une population très réduite de femelles.

Si l'on prend l'exemple d'un noyau de 200 femelles, avec 5 embryons par récolte et 40 p. 100 de taux de survie des embryons, l'application du schéma JJ 16 amène à choisir sur ascendance 12,5 taureaux par an. Ces taureaux sont issus euxmêmes de 12,5 taureaux et de 4,9 vaches. Les femelles de 1 an sélectionnées sur ascendance sont au nombre de 200 et sont issues de 12,5 taureaux et de 66 vaches. En ce qui concerne le schéma $\mathrm{A}$ correspondant, le nombre de taureaux pères est plus faible (3) mais ils sont issus d'une population de 43 vaches, celle qui a servi à fournir les 130 taureaux de testage annuels. Les femelles de 1 an sont issues de 3 pères et de 66 vaches.

\section{TABLEAU 7}

Augmentation annuelle du coefficient de consanguinité (p. 100) en fonction du type de schéma de transfert, de la taille du noyau, du nombre d'embryons transférables par récolte, du taux de survie des embryons.

Yearly increase of the inbreeding coefficient ( $p .100)$ according to transfer scheme, nucleus size, number of embryos per recovery, survival rate of the embryos.

\begin{tabular}{|c|c|c|c|c|c|c|c|c|}
\hline \multirow{2}{*}{$\begin{array}{c}\text { Taille } \\
\text { du } \\
\text { noyau }\end{array}$} & \multirow[b]{2}{*}{ Schéma } & Nombre d'embryons & \multicolumn{2}{|c|}{4} & \multicolumn{2}{|c|}{5} & \multicolumn{2}{|c|}{8} \\
\hline & & $\begin{array}{l}\text { Taux de survie } \\
\text { des embryons }\end{array}$ & $40 \%$ & $60 \%$ & $40 \%$ & $60 \%$ & $40 \%$ & $60 \%$ \\
\hline \multirow{5}{*}{100} & A & & - & 0,22 & 0,22 & 0,22 & 0,22 & 0,23 \\
\hline & B & & 一 & 0,17 & 0,17 & 0,17 & 0,17 & 0,19 \\
\hline & $\mathrm{B}^{\prime}$ & & - & 0,16 & 0,16 & 0,16 & 0,16 & 0,16 \\
\hline & JJ 16 & & - & 1,23 & 1,12 & 1,42 & 1,47 & 1,95 \\
\hline & JJ 32 & & 一 & 2,35 & 2,12 & 2,68 & 2,78 & 3,66 \\
\hline \multirow{5}{*}{200} & A & & 0,21 & 0,22 & 0,22 & 0,22 & 0,22 & 0.22 \\
\hline & B & & 0,16 & 0,16 & 0,16 & 0,16 & 0,16 & 0,17 \\
\hline & $\mathrm{B}^{\prime}$ & & 0,15 & 0,15 & 0,15 & 0,15 & 0,15 & 0,16 \\
\hline & JJ 16 & & 0,50 & 0,62 & 0,56 & 0,71 & 0,74 & 0,97 \\
\hline & JJ 32 & & 0,95 & 1,18 & 1,06 & 1,34 & 1,39 & 1,83 \\
\hline \multirow{5}{*}{400} & $\mathbf{A}$ & & 0,21 & 0,21 & 0,21 & 0,22 & 0,22 & 0,22 \\
\hline & B & & 0,15 & 0,15 & 0,15 & 0,16 & 0,16 & 0.16 \\
\hline & $\mathrm{B}^{\prime}$ & & 0,15 & 0,15 & 0,15 & 0,15 & 0,15 & 0,15 \\
\hline & JJ 16 & & 0,25 & 0,31 & 0,28 & 0,35 & 0,37 & 0,49 \\
\hline & JJ 32 & & 0,48 & 0,59 & 0,53 & 0,67 & 0,69 & 0,91 \\
\hline
\end{tabular}




\section{Vitesse d'obtention du régime asymptotique}

Cette dernière est évaluée en comparant d'une part les coefficients de régression du niveau génétique sur l'année (au cours de 3 périodes adjacentes de 10 ans) et d'autre part, le progrès génétique asymptotique calculé par la formule de RENDEL \& ROBERTSON.

Les niveaux génétiques initiaux des femelles du noyau sont considérés comme identiques aux niveaux génétiques des jeunes mâles contemporains destinés au testage. Ceci est en effet tout à fait réalisable, au moins dans le cas de tailles de noyau d'importance raisonnable.

Les valeurs obtenues sont remarquablement groupées. En raisonnant sur l'ensemble des paramètres envisagés (taille du noyau, type de schéma, nombre d'embryons, taux de survie, période de 10 ans), soit 51 valeurs, aucune n'est extérieure à l'intervalle $99-102$ p. 100 de la valeur asymptotique. On peut ainsi estimer que le régime asymptotique est pratiquement obtenu immédiatement. La succession très rapide des générations en est probablement la raison essentielle.

\section{Discussion}

\section{A. Progrès génétiques annuels}

\section{Evolution de la variance génétique}

Les progrès génétiques précédemment présentés sont très élevés. Sont-ils notoirement surévalués?

Il est indispensable de se poser cette question car toute sélection intensive entraîne à long terme une augmentation de la consanguinité et une réduction de la variabilité génétique. Si l'on prend en compte les algorithmes classiques, qui conviennent en fait à des populations non sélectionnées homologues en effectif, la réduction annuelle de variance génétique est peu sensible pour les schémas de transfert des types A, B, $B^{\prime}$ puisqu'elle se situe entre 0,15 et 0,22 p. 100 . Pour les Moer de type $\mathrm{JJ}$, le problème se pose plus nettement comme le signalent d'ailleurs Nicholas \& SMITH, puisque les réductions annuelles sont de l'ordre de 1 à 2 p. 100.

En fait, même dans le cas où le déterminisme génétique est simple (très grand nombre de loci non liés et additivité des effets des gènes), l'évolution de l'espérance de la variance génétique dans les populations sélectionnées à effectif limité est complexe et difficile à quantifier. En effet, interviennent pour la création de la variabilité génétique à la génération $\mathrm{g}$ :

1. La sélection des parents à la génération $\mathrm{g}-1$, qui présente donc une variance génétique moindre que celle de la génération $\mathrm{g}-1$. Par ailleurs, elle induit un déséquilibre de linkage artificiel (BuLMER, 1971) se transmettant partiellement à la génération suivante (il n'affecte pas en particulier la variance intrafamille).

2. Le coefficient vrai de parenté à l'intérieur d'une famille de même père et de même mère. 
3. L'échantillonnage des gamètes produisent la génération $\mathrm{g}$ parmi tous les gamètes possibles issus des parents sélectionnés à la génération $\mathrm{g}-1$.

Le premier point est développé en annexe 4, avec la transposition aux schémas étudiés, en négligeant les 2 autres phénomènes. Comme Bulmer (1971), MuEller \& JAMES (1983), nous nous sommes en outre placés dans le cas limite où les fréquences alléliques sont stables. Le second point a été traité par Foulley \& CHEVALET (1981), SorENSEN \& KenNEDY (1983) : le coefficient de parenté entre 2 pleins frères (sœurs) est supérieur à $1 / 4$ et la variance génétique intrafamille est inférieure à la moitié de la variance génétique initiale. Le facteur de correction nécessite de connaître les valeurs réelles de $F$ (ceux qui seraient fournis par le pedigree), qui mettent en œuvre non seulement les effectifs (comme dans les formules classiques) mais aussi les processus mêmes de sélection (RoBertson, 1961, 1977 ; BuRRows, 1984). La réduction supplémentaire de variance due à l'échantillonnage des gamètes ne fait intervenir que des processus aléatoires. Le coefficient de réduction est alors $1 / 2 \mathrm{~N}_{\mathrm{e}}, \mathrm{N}_{\mathrm{e}}$ étant calculé suivant les relations classiques (DempFle, 1975).

Dans ces conditions l'évolution asymptotique de la variance génétique n'est pas aisée à calculer car elle fait intervenir notamment l'évolution asymptotique vraie des coefficients de consanguinité. Un traitement correct de ce problème en ce qui concerne nos schémas nécessiterait par la suite des simulations aléatoires, avec calcul des coefficients $F$ pour chaque individu.

Cependant les exemples numériques traités par BuLMer montrent que les variances génétiques peuvent être réduites de façon très significative $(10$ ou $20 \mathrm{p} .100)$, en quelques générations, ce qui est sans commune mesure avec la décroissance asymptotique. Il paraît donc sage de prendre en considération ce phénomène de réduction immédiate de la variabilité génétique, dans les évaluations de progrès génétiques, en utilisant les approximations développées en annexe 4 et mentionnées précédem. ment.

Si l'on admet que le coefficient d'héritabilité de la production laitière est de 0,25 $(\sigma \mathrm{G}=0,50$ unité physique) avant toute sélection, le progrès génétique asymptotique obtenu avec le schéma classique passe alors de 0,226 à 0,177 écart-type génétique vrai (à l'équilibre) soit une réduction de $22 \mathrm{p}$. 100 , réduction très importante qui s'explique non seulement par la réduction de l'écart-type génétique mais également par la chute de précision des index de sélection. Si le coefficient d'héritabilité a été calculé pendant la phase de régime asymptotique du schéma classique, le coefficient d'héritabilité à l'équilibre est alors de $0,33(\sigma \mathrm{G}=0,61$ unité physique). Dans ce cas, le progrès génétique annuel calculé classiquement correspond à 0,239 unité du nouvel écart-type génétique, valeur qui passe à 0,186 quand on tient compte de l'effet Bulmer, soit une réduction de 22 p. 100 également. Toute cette série de valeurs met en lumière l'ampleur de l'incidence des déséquilibres de linkage induits tant par la réduction des progrès génétiques réellement possibles (de l'ordre de 20 p. 100) que par leur effet sur la signification des paramètres génétiques. En effet, dans notre cas, le progrès génétique vrai exprimé en unités physiques peut varier, à la limite, de 28 p. 100 suivant que les paramètres génétiques pris en considération sont évalués avant ou après le processus de sélection $\left(1^{\text {er }}\right.$ cas : 0,$177 ; 2^{\circ}$ cas : 0,186 $\times 0,61 / 0,50)$.

Les schémas de transfert ont été traités de la même manière et ont montré eux aussi une réduction importante du progrès génétique. Cependant, dans le cas des 
schémas $\mathrm{A}, \mathrm{B}, \mathbf{B}^{\prime}$ cette réduction est comparativement moindre que celle du schéma classique : leur efficacité exprimée en p. 100 de ce schéma, augmente d'environ 5 points (tabl. 8). La supériorité très nette du schéma $A$ apparaît alors (de l'ordre de 30 points d'écart en moyenne par rapport à la référence). Ce résultat tient au fait que dans ce schéma la pression de sélection est en moyenne plus faible sur les voies mère-fils et mère-fille et concerne des index de sélection en moyenne moins précis. Toutes ces constatations sur les classements relatifs des schémas sont pratiquement indépendantes de la signification de la mesure de l'héritabilité.

Dans le cas des schémas de type MoET, la pression de sélection est extrêmement élevée sur les voies mère-fils et mère-fille et surtout sur les voies père-fils et pèrefille. Cependant, elle a un retentissement plus faible sur les variances génétiques que les autres schémas, en raison de la faible précision des indices de sélection (coefficients de détermination de l'ordre de 0,10 à 0,12 ). De ce fait, la prise en considération des déséquilibres de linkage revalorise considérablement l'intérêt des MoET par rapport au schéma de référence $(+25$ points environ) et par rapport aux autres schémas de transfert $(+20$ points), comme cela a été d'ailleurs signalé à l'auteur par Sмiтн (communication personnelle).

\section{Calcul de la supériorité génétique des reproducteurs sélectionnés}

Même dans le cas où la variance génétique est bien connue, le calcul de ces valeurs est en toute rigueur complexe si les populations sélectionnées sont à la fois d'effectif limité, d'apparentement moyen important et réparties sur un petit nombre de familles (HILl, 1977, 1984). Les calculs exacts ont été effectués par cet auteur dans des cas particuliers mais aucune approximation générale simple n'a été encore proposée. Les biais de surévaluation présentés par le mode de calcul classique (population infinie sans apparentement) ou par l'approximation de BurRows (1972) (population finie sans apparentement) peuvent être importants.

Les conditions précédemment mentionnées concernent toutes les voies de transmission du progrès génétique de tous les schémas étudiés, particulièrement les voies père-fils et père-fille.

En ce qui concerne la voie père-fils du schéma classique, le calcul classique donne une supériorité de 1,99 unité d'écart-type génétique, celui de Burrows 1,93 et une première approximation, encore biaisée (HILl, 1976), 1,91. En fait, la véritable valeur pourrait se situer aux alentours de 1,80 (résultat de 500 simulations portant sur 100 taureaux issus de 3 pères, eux-mêmes tirés au hasard). En l'absence d'apparentement supposé, les simulations (500 également) permettent d'aboutir à une valeur proche de celle de Burrows $(1,94)$. En ce qui concerne la voie père-fils du schéma classique, les 5 valeurs correspondantes sont 1,$43 ; 1,42 ; 1,41 ; 1,33 ; 1,43$.

Dans ces conditions, la surévaluation du progrès génétique annuel obtenu par le schéma classique pourrait être de l'ordre de 5 p. 100 soit 0,01 écart-type génétique, en négligeant l'erreur faite sur les voies mère-fils et mère-fille.

Le biais effectué sur les schémas de transfert $\mathrm{A}, \mathrm{B}, \mathrm{B}^{\prime}$ est encore plus important. En conséquence, leur supériorité relative sur le schéma classique devrait être diminuée d'environ 2 à 3 points. Cette valeur est de l'ordre de grandeur de l'avantage (5 points) donné à ces schémas par la prise en compte des déséquilibres de linkage. Les 2 effets contradictoires, discutés aux paragraphes 1 et 2 , s'annulant pratiquement, il est 
TABLEaU 8

Progrès génétique asymptotique en p. 100 de la référence

après prise en considération des déséquilibres de linkage induits par la sélection.

Asymptotic genetic gains (p. 100 reference)

taking into account the linkage disequilibrium induced by selection.

\begin{tabular}{|c|c|c|c|c|c|c|c|c|}
\hline \multirow{2}{*}{$\begin{array}{c}\text { Taille } \\
\text { du } \\
\text { noyau }\end{array}$} & \multirow[b]{2}{*}{ Schéma } & $\begin{array}{l}\text { Nombre d'embryons } \\
\text { par récolte }\end{array}$ & \multicolumn{2}{|c|}{4} & \multicolumn{2}{|c|}{5} & \multicolumn{2}{|c|}{8} \\
\hline & & $\begin{array}{l}\text { Taux de survie } \\
\text { des embryons }\end{array}$ & $40 \%$ & $60 \%$ & $40 \%$ & $60 \%$ & $40 \%$ & $60 \%$ \\
\hline \multirow{5}{*}{100} & A & & - & $\begin{array}{l}121 \\
119 \\
\end{array}$ & $\begin{array}{l}118 \\
116 \\
\end{array}$ & $\begin{array}{l}125 \\
123 \\
\end{array}$ & $\begin{array}{l}125 \\
123 \\
\end{array}$ & $\begin{array}{l}131 \\
129 \\
\end{array}$ \\
\hline & B & & - & $\begin{array}{l}99 \\
97\end{array}$ & $\begin{array}{l}95 \\
93\end{array}$ & $\begin{array}{l}102 \\
100\end{array}$ & $\begin{array}{l}103 \\
101\end{array}$ & $\begin{array}{l}108 \\
106\end{array}$ \\
\hline & $B^{\prime}$ & & - & $\begin{array}{l}106 \\
104\end{array}$ & $\begin{array}{l}103 \\
101\end{array}$ & $\begin{array}{l}110 \\
108\end{array}$ & $\begin{array}{l}111 \\
110\end{array}$ & $\begin{array}{l}117 \\
116\end{array}$ \\
\hline & JJ 16 & & - & $\begin{array}{l}136 \\
137\end{array}$ & $\begin{array}{l}125 \\
126\end{array}$ & $\begin{array}{l}148 \\
149\end{array}$ & $\begin{array}{l}151 \\
152\end{array}$ & $\begin{array}{l}170 \\
170\end{array}$ \\
\hline & JJ 32 & & - & $\begin{array}{l}147 \\
147\end{array}$ & $\begin{array}{l}137 \\
137\end{array}$ & $\begin{array}{l}158 \\
158\end{array}$ & $\begin{array}{l}161 \\
161\end{array}$ & $\begin{array}{l}177 \\
177\end{array}$ \\
\hline \multirow{5}{*}{200} & $\overline{\mathrm{A}}$ & & $\begin{array}{l}120 \\
119\end{array}$ & $\begin{array}{l}127 \\
125\end{array}$ & $\begin{array}{l}124 \\
122\end{array}$ & $\begin{array}{l}130 \\
128\end{array}$ & $\begin{array}{l}130 \\
129\end{array}$ & $\begin{array}{l}136 \\
134\end{array}$ \\
\hline & B & & $\begin{array}{l}98 \\
96\end{array}$ & $\begin{array}{l}104 \\
102\end{array}$ & $\begin{array}{l}101 \\
100\end{array}$ & $\begin{array}{l}107 \\
105\end{array}$ & $\begin{array}{l}107 \\
106\end{array}$ & $\begin{array}{l}112 \\
111\end{array}$ \\
\hline & $\mathbf{B}^{\prime}$ & & $\begin{array}{l}102 \\
101\end{array}$ & $\begin{array}{l}110 \\
108\end{array}$ & $\begin{array}{l}106 \\
105\end{array}$ & $\begin{array}{l}113 \\
111\end{array}$ & $\begin{array}{l}113 \\
112\end{array}$ & $\begin{array}{l}119 \\
118\end{array}$ \\
\hline & JJ 16 & & $\begin{array}{l}110 \\
112\end{array}$ & $\begin{array}{l}136 \\
137\end{array}$ & $\begin{array}{l}125 \\
126\end{array}$ & $\begin{array}{l}148 \\
149\end{array}$ & $\begin{array}{l}151 \\
152\end{array}$ & $\begin{array}{l}170 \\
170\end{array}$ \\
\hline & JJ 32 & & $\begin{array}{l}122 \\
123\end{array}$ & $\begin{array}{l}147 \\
147\end{array}$ & $\begin{array}{l}137 \\
137\end{array}$ & $\begin{array}{l}158 \\
158\end{array}$ & $\begin{array}{l}161 \\
161\end{array}$ & $\begin{array}{l}178 \\
177\end{array}$ \\
\hline \multirow{5}{*}{400} & A & & $\begin{array}{l}125 \\
124\end{array}$ & $\begin{array}{l}132 \\
130\end{array}$ & $\begin{array}{l}129 \\
127\end{array}$ & $\begin{array}{l}134 \\
132\end{array}$ & $\begin{array}{l}135 \\
133\end{array}$ & $\begin{array}{l}140 \\
138\end{array}$ \\
\hline & B & & $\begin{array}{l}103 \\
101\end{array}$ & $\begin{array}{l}108 \\
106\end{array}$ & $\begin{array}{l}106 \\
105\end{array}$ & $\begin{array}{l}110 \\
109\end{array}$ & $\begin{array}{l}111 \\
110\end{array}$ & $\begin{array}{l}115 \\
114\end{array}$ \\
\hline & $\mathbf{B}^{\prime}$ & & $\begin{array}{l}106 \\
105\end{array}$ & $\begin{array}{l}113 \\
111\end{array}$ & $\begin{array}{l}110 \\
108\end{array}$ & $\begin{array}{l}116 \\
115\end{array}$ & $\begin{array}{l}117 \\
115\end{array}$ & $\begin{array}{l}121 \\
121\end{array}$ \\
\hline & JJ 16 & & $\begin{array}{l}110 \\
112\end{array}$ & $\begin{array}{l}136 \\
137\end{array}$ & $\begin{array}{l}125 \\
126\end{array}$ & $\begin{array}{l}148 \\
149\end{array}$ & $\begin{array}{l}151 \\
152\end{array}$ & $\begin{array}{l}170 \\
170\end{array}$ \\
\hline & JJ 32 & & $\begin{array}{l}122 \\
123\end{array}$ & $\begin{array}{l}147 \\
147\end{array}$ & $\begin{array}{l}137 \\
137\end{array}$ & $\begin{array}{l}158 \\
158\end{array}$ & $\begin{array}{l}161 \\
161\end{array}$ & $\begin{array}{l}178 \\
177\end{array}$ \\
\hline
\end{tabular}

$1^{\text {r }}$ ligne : Le coefficient d'héritabilité de 0,25 a été calculé avant toute sélection (valeur finale : 0,18 ). 1st line : The heritabiiity $(0.25)$ was evaluated before any selection (eventual value : 10.18).

$2^{\circ}$ ligne : Le coefficient d'héritabilité de 0,25 a été calculé après instauration d'un nouvel équilibre (valeur initiale : 0,33).

2nd line : The heritability (0.25) was evaluated after the new steady state (initial value: 0.33 ). 
possible de penser qu'au moins à court terme les valeurs du tableau 4 présentant la supériorité relative des schémas de transfert, avec les simplifications désormais classiques, ne devraient pas être trop éloignées de la réalité.

\section{B. Evolution des coefficients de consanguinité}

Comme signalé précédemment, un traitement correct du problème nécessiterait l'utilisation de la simulation aléatoire.

L'utilisation de l'algorithme de Burrows (1984) pour le premier cycle de sélection des femelles du noyau amène à des résultats très éloquents en ce qui concerne la probabilité de même origine paternelle des femelles sélectionnées (tabl. 9). Ils montrent clairement que dans les schémas $A, B, B^{\prime}$, il est effectivement plus sage de sélectionner les femelles sur performances et non sur index global, cumulant performances et index du père (Colleau \& Poutous, 1973).

Le calcul de Burrow effectué au cours du premier cycle de sélection des mâles du noyau pour évaluer la probabilité que 2 mâles sélectionnés aient la même origine paternelle permet d'obtenir une valeur bien supérieure à 0,33 c'est-à-dire 0,48 . Qualitativement, on peut penser que l'écart entre ces 2 valeurs n'est pas constant mais diminue au fur et à mesure que la variance génétique se réduit mais ce résultat obtenu en début de sélection montre bien les biais encourus par les évaluations classiques d'augmentation de la consanguinité dans les schémas de sélection.

\section{Ouverture du noyau de sélection}

Ce noyau peut théoriquement voir son efficacité augmenter s'il reçoit certaines femelles de la population générale (JAMES, 1977). L'étude numérique devrait être entreprise ultérieurement. Il est néanmoins possible de penser que les taux optima d'ouverture risquent de diminuer au cours du temps, en raison de la progression très rapide des niveaux génétiques dans le noyau. Par ailleurs, ces taux sont sans doute faibles en raison de la grande supériorité génétique initiale du noyau sur l'ensemble de la population femelle.

Plus intéressante est la perspective de l'utilisation de la supériorité éventuelle d'une population extérieure, par exemple par importation d'embryons aboutissant à une introduction dans le noyau de mâles et de femelles extérieurs. La procédure pratique optimale nécessite une étude spécifique.

Par ailleurs, si plusieurs noyaux de sélection fonctionnent simultanément dans la même race, il n'est pas interdit de penser qu'ils pourront s'échanger des reproducteurs, notamment pour préserver la variabilité génétique, ou même pratiquement fusionner pour augmenter la capacité de testage global. Là encore, les études numériques correspondantes mettent en jeu de nombreux paramètres possibles, et il n'est pas question ici de traiter cette nouvelle possibilité.

\section{Introduction d'autres caractères que la production laitière}

D'autres caractères que la production laitière peuvent être introduits, à condition toutefois qu'ils n'entraînent pas un ralentissement de l'intervalle de génération, ce 
qui exclut pratiquement la prise en considération des caractères de «fitness » (reproduction et résistance aux maladies) observables pendant la vie productive de la femelle. Ceci est valable pour tous les schémas comparés, MoET compris. Les précautions proposées pour les schémas $\mathrm{A}, \mathrm{B}$ et $\mathbf{B}^{\prime}$ ne permettent de prendre en considération que la variabilité génétique de la reproduction avant le premier vêlage, ce qui n'est qu'une partie du problème global de la reproduction femelle.

\section{Tableau 9}

Influence de la méthode de sélection sur la probabilité

pour que 2 femelles sélectionnées soient issues du même père (schémas $A, B, B^{\prime}$ ).

Influence of the selection method (own performance or performance + sire evaluation) on the probability that 2 selected females are sired by the same bull.

\begin{tabular}{|c|c|c|c|c|}
\hline \multirow{2}{*}{$\begin{array}{c}\text { Taille } \\
\text { du noyau }\end{array}$} & \multirow{2}{*}{$\begin{array}{c}\text { Pression } \\
\text { de sélection }\end{array}$} & \multicolumn{3}{|c|}{ Méthode de sélection } \\
\hline & & $\begin{array}{c}\text { Tirage } \\
\text { au hasard }\end{array}$ & $\begin{array}{c}\text { sur } \\
\text { performance }\end{array}$ & $\begin{array}{c}\text { sur index } \\
\text { global }\end{array}$ \\
\hline 100 & $\begin{array}{l}10 \% \\
20 \% \\
30 \% \\
40 \% \\
60 \% \\
80 \%\end{array}$ & 0,327 & $\begin{array}{l}0,350 \\
0,340 \\
0,335 \\
0,333 \\
0,330 \\
0,328\end{array}$ & $\begin{array}{l}0,483 \\
0,419 \\
0,388 \\
0,369 \\
0,346 \\
0,332\end{array}$ \\
\hline 200 & $\begin{array}{l}10 \% \\
20 \% \\
30 \% \\
40 \% \\
60 \% \\
80 \%\end{array}$ & 0,330 & $\begin{array}{l}0,354 \\
0,343 \\
0,339 \\
0,336 \\
0,333 \\
0,331\end{array}$ & $\begin{array}{l}0,489 \\
0,423 \\
0,392 \\
0,373 \\
0,349 \\
0,336\end{array}$ \\
\hline 400 & $\begin{array}{l}10 \% \\
20 \% \\
30 \% \\
40 \% \\
60 \% \\
80 \%\end{array}$ & 0,332 & $\begin{array}{l}0,356 \\
0,345 \\
0,341 \\
0,338 \\
0,335 \\
0,333\end{array}$ & $\begin{array}{l}0,491 \\
0,425 \\
0,394 \\
0,374 \\
0,351 \\
0,338\end{array}$ \\
\hline
\end{tabular}

Une série importante de caractères secondaires est celle concernant les performances de croissance et de conformation bouchère. La prise en considération effective de ces caractères dans les noyaux de sélection est nettement moins aisée que pour les schémas classiques, tout au moins dans le cas du schéma $A$, qui est celui qui nous semble le plus performant dans le cadre de la contrainte d'organisation collective mentionnée au tout début. 
Il serait alors nécessaire de contrôler tous les mâles dans des conditions rigoureusement définies (observation d'un protocole de contrôle individuel), c'est-à-dire en clair de les récupérer tous après la naissance et de les regrouper en station ce qui constitue une opération coûteuse. Les femelles pourraient être éventuellement contrôlées en ferme, mais la précision serait moindre et par ailleurs, il faudrait pouvoir contraindre les éleveurs à éliminer les femelles déficientes sur ces caractères secondaires. Et finalement, tout effort de sélection dans ce domaine augmenterait d'autant la taille du noyau, le nombre de veaux mâles à conserver, le nombre de transferts à effectuer, c'est-à-dire entraînerait des coûts supplémentaires importants, bien plus importants en tout cas que dans les schémas classiques (Ducroce, 1984).

\section{Conclusion}

Les analyses numériques précédemment développées permettent de conclure que les noyaux de sélection utilisant le transfert accéléré d'embryons (type $A$ ), dans un cadre collectif défini comme condition de base, sont potentiellement efficaces pour l'amélioration génétique de la production laitière. En effet, ils induisent un progrès génétique d'au moins 20 p. 100 supérieur à celui d'un schéma classique très bien mené. Cette valeur est obtenue avec les paramètres techniques actuels, caractérisant la superovulation et le transfert des embryons, alors qu'elle ne pourrait être obtenue qu'avec une amélioration drastique des techniques, si le transfert n'était utilisé que dans le cadre des schémas classiques (notamment sans changement de l'intervalle de génération).

Les 2 raisons de l'efficacité de ce type de noyau sont le cumul de 2 facteurs très favorables :

- diminution de l'intervalle de génération sur les voies mère-fils et mère-fille ;

- sélection des pères sur des index de sélection précis, exactement comme dans les schémas classiques.

Dans la réalité, la conduite des noyaux de sélection pourrait être nettement plus complexe que celle décrite dans ce texte. Le noyau peut être ouvert à la population générale, à d'autres noyaux, à des populations exotiques. La sélection peut porter sur d'autres caractères que la production laitière, bien que nous pensions que leur prise en considération risque d'être délicate.

Les perspectives paraissent suffisamment intéressantes pour aborder par la suite des études tenant compte de ces possibilités et également de la dimension économique correspondante.

\section{Remerciements}

Nous remercions les lecteurs anonymes mandatés par la revue pour leur examen critique approfondi du manuscrit. 


\section{Annexes}

\section{A. Calcul de la variance des tailles de famille} dans le cas du schéma classique de référence

La relation de HiLl (1979) s'applique en faisant $\mathrm{m}=$ nombre de mâles par génération $=$ nombre de mâles par année $\times$ intervalle de génération $=100 \mathrm{~L}$. De la même manière $\mathrm{f}=$ nombre de femelles par génération $=52000 \mathrm{~L}$.

Le calcul de la variance des tailles de famille d'origine paternelle est compliqué par le fait que les pères n'ont pas la même probabilité a priori d'avoir des descendants à la génération suivante. On peut estimer qu'ils se répartissent en groupes différents à l'intérieur desquels les probabilités sont les mêmes. Si l'on désigne par i l'indice groupe, $T_{i}$ pères auront au total $D_{i}$ descendants. Nous ferons l'approximation que pour un i donné, $T_{i}$ et $D_{i}$ ne sont pas des variables aléatoires (effectifs par groupe fixés). La variance totale de la taille de famille d se déduit alors simplement et classiquement en fonction des sommes de carrés entre groupes et des sommes de carrés intragroupes, ces derniers se déduisant immédiatement de la variance d'une loi binomiale :

soit :

$$
\left(D_{i} / T_{i}\right)\left(1-1 / T_{i}\right)
$$

$$
v(d)=\left(1 / \sum_{i} T_{i}\right)\left[\sum_{i} T_{i}\left(\left(D_{i} / T_{i}\right)-\bar{D}\right)^{2}+\sum_{i} D_{i}\left(1-1 / T_{i}\right)\right]
$$

Pour la voie père-fils :

$$
\begin{array}{lll}
\mathrm{T}=100 & \mathrm{~T}_{1}=3 & \mathrm{~T}_{2}=97 \\
\mathrm{D}=100 & \mathrm{D}_{1}=100 & \mathrm{D}_{2}=0
\end{array}
$$

et $\mathrm{v}(\mathrm{d})=\sigma_{1}^{2}=33$

Pour la voie père-fille :

$$
\begin{array}{lll}
\mathrm{T}=100 & \mathrm{~T}_{1}=13 & \mathrm{~T}_{2}=87 \\
\mathrm{D}=52000 & \mathrm{D}_{1}=47476 & \mathrm{D}_{2}=4524
\end{array}
$$

et $v(d)=\sigma_{2}^{2}=2530000$

En ce qui concerne les ascendants femelles, on considère que les probabilites a priori sont identiques pour chaque femelle. On est alors ramené à la loi binomiale et si le nombre de femelles est grand, on aura :

$$
\sigma_{3}^{2} \# \mathrm{~m} / \mathrm{f} \quad \sigma_{4}^{2} \# 1
$$

Le calcul des covariances entre tailles de famille est nécessaire pour les voies père-fils et père-fille. On a alors dans ce cas 3 groupes de pères :
$T=100$
$\mathrm{T}_{1}=3$
$\mathrm{T}_{2}=10$
$\mathrm{T}_{3}=87$
$\mathrm{D}=100$
$\mathrm{D}_{1}=100$
$\mathrm{D}_{2}=0$
$\mathrm{D}_{3}=0$
$\mathrm{D}^{\prime}=52000$
$\mathrm{D}^{\prime}{ }_{1}=10956$
$\mathrm{D}_{2}^{\prime}=36520$
$\mathrm{D}_{3}^{\prime}=4524$ 
Le calcul des covariances s'établit à partir des sommes de coproduits entre groupes pour la variable $d$ (taille de la descendance chez les mâles) et pour la variable $d^{\prime}$ (taille de la descendance chez les femelles);

$$
\operatorname{Cov}\left(d, d^{\prime}\right)=\left[\sum_{i} T_{i}\left(\left(D_{i} / T_{i}\right)-\bar{D}\right)\left(\left(D_{i}^{\prime} / T_{i}\right)-\bar{D}^{\prime}\right)\right] / T
$$

On trouve alors que $\operatorname{Cov}(1,2)=3132$.

On a par ailleurs $\operatorname{Cov}(3,4)=0$, puisque les probabilités a priori ne diffèrent pas pour les femelles.

\section{B. Optimisation de schémas $B$ et $B^{\prime}$}

Cette optimisation est effectuée algébriquement en utilisant la méthode de NewTON-RAphson. Si la fonction à optimiser est notée $y(t)$, la solution est $Y=y(\tilde{t})$ où $\tilde{\mathrm{t}}$ est la valeur de convergence de l'algorithme itératif :

$$
t_{a+1}=t_{a}-y_{t_{a}}^{\prime} / y^{\prime \prime} t_{a}
$$

\section{a) Optimisation du schéma $B$}

$\Delta G \quad$ est le progrès génétique annuel calculé par la formule de Rendel \& RoBERTSON et exprimé en unité d'écart type génétique.

$\mathrm{N} \quad$ est la taille du noyau : $\mathrm{n}_{1}+\mathrm{n}_{2}$.

$\mathrm{n}_{1} \quad$ est le nombre de femelles de renouvellement issues de transfert.

$\mathrm{n}_{2} \quad$ est le nombre de femelles normales de renouvellement.

$k_{1}$ et $k_{2} \quad$ sont respectivement les nombres de génisses de transfert et de génisses normales utilisables, obtenus pour une femelle donneuse.

$\mathrm{N} * \quad$ est le nombre de mères potentielles (nombre de vaches en première lactation ayant satisfait aux diverses exigences de reproduction).

On a $\mathrm{N}^{*} / \mathrm{N}=\mathrm{c}=$ constante $(0<\mathrm{c}<1)$, ne dépendant que des seules règles de démographie et de reproduction retenues.

$\mathrm{p}_{41}$ et $\mathrm{p}_{42}$ sont les pressions de sélection effectuées sur les mères des $n_{1}$ et $n_{2}$ génisses respectivement.

$i_{41}$ et $i_{42}$ sont les intensités de sélection correspondantes.

$\mathbf{L}_{41}$ et $\mathbf{L}_{\mathbf{4} 2}$ sont les intervalles de génération correspondants.

$I_{1}=I_{2}=$ supériorité génétique sur les voies père-fils et père-fille.

$\mathrm{I}_{3}=\quad$ supériorité génétique sur la voie mère-fils.

$\mathbf{L}_{1}, \mathbf{L}_{2}, \mathbf{L}_{3} \quad$ les intervalles de génération correspondant à ces trois voies.

$\Delta G$ s'exprime alors en unité d'écart-type génétique par :

$$
\Delta G=\frac{I_{1}+I_{2}+I_{3}+h N^{-1}\left(n_{1} i_{41}+n_{2} i_{42}\right)}{L_{1}+L_{2}+L_{3}+N^{-1}\left(n_{1} L_{41}+n_{2} L_{42}\right)}
$$


Si l'on prend comme variable de dérivation $\mathrm{p}_{41}$, on $\mathrm{a}$ :

$$
\begin{gathered}
\mathrm{n}_{1}=\mathrm{p}_{41} \mathrm{k}_{1} \mathrm{~N}^{*} \quad \mathrm{n}_{2}=\mathrm{N}-\mathrm{p}_{41} \mathrm{k}_{1} \mathrm{~N}^{*} \\
\Delta \mathrm{G}=\frac{\mathrm{N}\left(\mathrm{I}_{1}+\mathrm{I}_{2}+\mathrm{I}_{3}\right)+\mathrm{hN}^{*}\left(\mathrm{p}_{41} \mathrm{k}_{1} \mathrm{i}_{41}+\mathrm{p}_{42} \mathrm{k}_{2} \mathrm{i}_{42}\right)}{\mathrm{N}\left(\mathrm{L}_{1}+\mathrm{L}_{2}+\mathrm{L}_{3}\right)+\mathrm{k}_{1} \mathrm{~N}^{*} \mathrm{p}_{41}\left(\mathrm{~L}_{41}-\mathrm{L}_{42}\right)} \\
=\frac{\alpha+\mathrm{hN} \mathrm{N}^{*}\left(\mathrm{p}_{41} \mathrm{k}_{1} \mathrm{i}_{41}+\mathrm{p}_{42} \mathrm{k}_{2} \mathrm{i}_{42}\right)}{\beta+\gamma \mathrm{p}_{41}}=\frac{\mathrm{u}}{\mathrm{v}}
\end{gathered}
$$

Le calcul des dérivées première et seconde de $\Delta G$ suppose le calcul des dérivées première et seconde de $\mathrm{u}$ et de $\mathrm{v}$.

En ce qui concerne le calcul de $\mathbf{u}^{\prime}$, on calcule :

$$
\frac{\delta\left(p_{41} i_{41}\right)}{\delta p_{41}}=p_{41} \cdot \frac{\delta i_{41}}{\delta p_{41}}+i_{41}=p_{41} \frac{x_{41}-i_{41}}{p_{41}}+i_{41}=x_{41}
$$

en désignant par $\mathrm{x}$ la valeur de la variable normale réduite au point de troncature.

$$
\frac{\delta\left(\mathrm{p}_{42} \mathrm{i}_{42}\right)}{\delta \mathrm{p}_{41}}=\frac{\delta\left(\mathrm{p}_{42} \mathrm{i}_{42}\right)}{\delta \mathrm{p}_{42}} \cdot \frac{\delta \mathrm{p}_{42}}{\delta \mathrm{p}_{41}}=-\frac{\mathrm{k}_{1}}{\mathrm{k}_{2}} \mathrm{x}_{42}
$$

soit :

$$
\begin{gathered}
\mathrm{u}^{\prime}=\mathrm{hk}_{1} \mathrm{~N}^{*}\left(\mathrm{x}_{41}-\mathrm{x}_{42}\right) \\
\frac{\delta \mathrm{x}_{41}}{\delta \mathrm{p}_{41}}=-\sqrt{2 \pi} \exp \left[\mathrm{x}_{41}^{2} / 2\right] \\
\frac{\delta \mathrm{x}_{42}}{\delta \mathrm{p}_{41}}=\frac{\delta \mathrm{x}_{42}}{\delta \mathrm{p}_{42}} \cdot \frac{\delta \mathrm{p}_{42}}{\delta \mathrm{p}_{41}}=\left(\mathrm{k}_{1} \sqrt{2 \pi} \cdot \exp \left[\mathrm{x}_{42}^{2} / 2\right]\right) / \mathrm{k}_{2}
\end{gathered}
$$

soit :

$$
\mathrm{u}^{\prime \prime}=-\mathrm{hk}_{1} \mathrm{~N}^{*} \sqrt{2 \pi}\left(\exp \left[\mathrm{x}_{41}^{2} / 2\right]+\left(\mathrm{k}_{1} / \mathrm{k}_{2}\right) \exp \left[\mathrm{x}_{42}^{2} / 2\right]\right)
$$

Par ailleurs :

$$
\mathbf{v}^{\prime}=\gamma \quad \mathbf{v}^{\prime \prime}=0
$$

b) Optimisation du schéma $B^{\prime}$

Ici le nombre de mères potentielles $\mathrm{N}^{*}$ est une variable. L'effectif correspondant du noyau de jeunes génisses est $\mathbf{N}=\mathrm{N}^{*} / \mathrm{c}$.

Les paramètres de la voie mère-fils deviennent variables, la pression de sélection le long de cette voie est $\mathrm{p}_{3}=130 / \mathrm{k}_{1} \mathrm{~N}^{*}$, l'intensité de sélection est $\mathrm{i}_{3}$ et l'intervalle de génération est $\mathrm{L}_{3}=\mathrm{L}_{41}$.

En revanche, $\mathrm{n}_{1}$ devient constant. 


$$
\Delta G=\frac{I_{1}+I_{2}+h N-1\left(N_{3}+n_{41} i_{41}+n_{42} i_{42}\right)}{L_{1}+L_{2}+L_{41}+N^{-1}\left(n_{41} L_{41}+n_{42} L_{42}\right)}
$$

On prend comme variable de dérivation le rapport $\mathrm{p}_{41}^{*}=\mathrm{n}_{1} / \mathrm{N}$ qui amène à des expressions plus simples que la pression de sélection $\mathrm{p}_{41}$, précédemment utilisée.

On a :

$$
\begin{aligned}
& \mathrm{p}_{41}=\mathrm{cp}_{41}^{*} / \mathrm{k}_{1} \quad \mathrm{p}_{42}=\mathrm{c}\left(1-\mathrm{p}_{41}^{*}\right) / \mathrm{k}_{2} \\
& \mathrm{p}_{3}=130 \mathrm{cp}_{41}^{*} / \mathrm{k}_{1} \mathrm{n}_{41}
\end{aligned}
$$

On a donc à optimiser la fonction :

$$
\begin{aligned}
\Delta G= & \frac{I_{1}+I_{2}+h\left(i_{3}+p_{41}^{*} i_{41}+\left(1-p_{41}^{*}\right) i_{42}\right)}{L_{1}+L_{2}+L_{41}+L_{42}+p_{41}^{*}\left(L_{41}-L_{42}\right)} \\
= & \frac{\alpha^{*}+h i_{3}+h p_{41}^{*} i_{41}+h\left(1-p_{41}^{*}\right) i_{42}}{\beta^{*}+\gamma^{*} p_{41}^{*}}=\frac{u}{v}
\end{aligned}
$$

Le calcul de $\mathbf{u}^{\prime}$ permet d'aboutir à :

Celui de u", à :

$$
\mathrm{u}^{\prime}=\mathrm{x}_{41}-\mathrm{x}_{42}+\left(\mathrm{x}_{3}-\mathrm{i}_{3}\right) / \mathrm{p}_{41}^{*}
$$

Par ailleurs :

$$
\begin{aligned}
& \mathrm{u}^{\prime \prime}=-c \sqrt{2 \pi}\left(\mathrm{k}_{1}^{-1} \exp \left[\mathrm{x}_{41}^{2} / 2\right]+\mathrm{k}_{2}^{-1} \exp \left[\mathrm{x}_{42}^{2} / 2\right]\right. \\
& \left.+\left(130 / \mathrm{p}_{41}^{*} \mathrm{n}_{1} \mathrm{k}_{1}\right) \exp \left[\mathrm{x}_{3}^{2} / 2\right]\right)-2\left(\mathrm{x}_{3}-\mathrm{i}_{3}\right) / \mathrm{p}_{41}^{* 2}
\end{aligned}
$$

$$
\mathrm{v}^{\prime}=\gamma^{\prime} \quad \mathrm{v}^{\prime \prime}=0
$$

C. Calcul de l'augmentation annuelle asymptotique du coefficient de consanguinité

Hrll (1979) a montré que l'évolution asymptotique par génération des coefficients de consanguinité dans une population à générations chevauchantes est la même que celle d'une population à générations séparées et ayant les mêmes effectifs de reproducteurs par génération.

Si l'on veut calculer le coefficient de consanguinité $F_{g}$ à la génération $g$ dans la population équivalente à générations séparées, on évalue la probabilité de 3 situations possibles.

a) Les conjoints à la génération $\mathrm{g}-1$ sont issus des mêmes parents à la génération $\mathrm{g}-2$.

- Probabilité $=$ Probabilité à la génération $\mathrm{g}$ de tirer la même mère $(1 / \mathrm{f})$ $\times$ Probabilité conditionnelle de tirer le même père $(1 / 2)$.

- Parenté $=\left(1+\mathrm{F}_{\mathrm{g}-2}+2 \mathrm{~F}_{\mathrm{g}-1}\right) / 4$. 
B) Les conjoints ont un seul parent en commun à la génération $\mathrm{g}-2$.

- Probabilité $=$ Probabilité à la génération $g$ de tirer la même mère $(1 / f) \times$ Probabilité de tirer un père différent $(1 / 2)$ + Probabilité de tirer le même père $(1 / \mathrm{m}) \times$ Probabilité de tirer une mère différente $(1-1 / \mathrm{f})$.

- Parenté $=\left(1+\mathrm{F}_{\mathrm{g}-2}+6 \mathrm{~F}_{\mathrm{g}-1}\right) / 8$.

$\gamma)$ Les conjoints n'ont pas de parents communs à la génération $\mathrm{g}-2$.

— Probabilité $=$ Probabilité à la génération $\mathrm{g}$ de tirer une mère différente $(1-1 / \mathrm{f}) \times$ Probabilité de tirer un père différent $(1-1 / \mathrm{m})$.

- Parenté $=\mathrm{F}_{\mathrm{g}-1}$.

La relation de récurrence devient alors :

avec :

$$
F_{g}=F_{g-1}+\alpha\left(1-2 F_{g-1}+F_{g-2}\right)
$$

$$
\alpha=\left(3 \mathrm{~m}_{\mathrm{a}}+2 \mathrm{f}_{\mathrm{a}}-2\right) / 16 \mathrm{~m}_{\mathrm{a}} \mathrm{f}_{\mathrm{a}}
$$

On peut montrer que dans ce cas, l'augmentation du coefficient de consanguinité $\Delta \mathrm{F}_{\mathrm{g}}=\left(\mathrm{F}_{\mathrm{g}}-\mathrm{F}_{\mathrm{g}-1}\right) / 1-\mathrm{F}_{\mathrm{g}-1}$ tend asymptotiquement vers $\alpha(\mathrm{LI}, 1955, \mathrm{p} .210)$. $\Delta \mathrm{F}$ est :

En revenant à l'augmentation annuelle, et non par génération, la limite de

$$
\left(3 \mathrm{~m}_{\mathrm{a}} \mathrm{L}+2 \mathrm{f}_{\mathrm{a}} \mathrm{L}-2\right) / 16 \mathrm{~L}^{3} \mathrm{~m}_{\mathrm{a}} \mathrm{f}_{\mathrm{a}} \#\left(3 \mathrm{~m}_{\mathrm{a}}+2 \mathrm{f}_{\mathrm{a}}\right) / 16 \mathrm{~L}^{2} \mathrm{~m}_{\mathrm{a}} \mathrm{f}_{\mathrm{a}}
$$

en désignant par ma et fa les effectifs annuels des mâles et des femelles.

Dans tous les schémas envisagés, une complication supplémentaire provient du fait que la probabilité de tirer la même mère n'est pas la même suivant qu'il s'agit de la voie mère-fils ( $f_{\mathrm{a} 3}$ mères différentes) ou mère-filles ( $f_{\mathrm{a} 4}$ mères différentes), où $f_{a 3}$ est un sous-ensemble de $f_{a 4}$.

La probabilité de tirer la même mère pour 2 individus différents est :

soit :

$$
\begin{gathered}
1 / f_{a}=\underset{(\delta, \delta)}{\left(1 / 4 f_{a 3}\right)}+\left(1 / 2 f_{a 3}\right)\left(f_{a 3} / f_{a 4}\right)+1 / 4 f_{a 4} \\
(\delta, \&)
\end{gathered}
$$

$$
f_{a}=4 f_{a 3} f_{a 4} /\left(f_{a 4}+3 f_{a 3}\right)
$$

\section{Réduction de la variance génétique par déséquilibre de linkage induit}

\section{a) Généralités}

L'hypothèse effectuée pour les calculs est la même que celle adoptée par BuLMER : la sélection n'affecte pas les fréquences alléliques (ce qui suppose un très grand nombre de loci et également un très grand nombre de reproducteurs). Par ailleurs, on suppose qu'il n'y a pas de linkage vrai (taux de recombinaison $=0,5$ pour tous les couples de loci).

Pour tous les schémas envisagés, il est nécessaire de tenir compte du fait que les mâles et les femelles contemporains peuvent ne pas avoir le même niveau génétique. Les variances génétiques à prendre en considération sont dans ces conditions les varianoes intra sexe, puisque la variance entre sexes n'est pas mise à profit par la sélection. 
Pour un sexe donné, on a alors :

variance génétique $=1 / 4$ (variance génétique des pères correspondants) $+1 / 4$ (variance génétique des mères correspondantes) + variance intra famille de même père et de même mère.

Cette dernière variance est par ailleurs constante et égale à la moitié de la variance génétique initiale, dans le cadre des hypothèses effectuées.

La variance génétique d'un ensemble d'animaux sélectionnés, dans l'hypothèse simplificatrice d'une conservation de la normalité des distributions à chaque génération, est égale à $\mathrm{V}_{\mathrm{A}}(1+\mathrm{K} . \mathrm{CD})$ où $\mathrm{V}_{\mathrm{A}}$ est la variance génétique de la population d'où ils sont tirés, $C D$ le coefficient de détermination de l'index utilisé, et $\mathrm{K}$ la réduction de variance des index, $K=-\mathrm{i}(\mathrm{i}-\mathrm{x})$ où $\mathrm{i}$ est la différentielle de sélection en unité d'écart-type d'index et $x$ la position du seuil de troncature dans la même unité.

b) Schéma classique de référence

A l'équilibre, on a :

$$
\begin{aligned}
& \mathrm{V}_{\mathrm{AM}}= \underset{\text { (voie père-fils) }}{1 / 4} \mathrm{~V}_{\mathrm{AM}}\left(1+\mathrm{K}_{1} \mathrm{CD}_{1}\right)+\underset{\text { (voie mère-fils) }}{1 / 4 \mathrm{~V}_{\mathrm{AF}}\left(1+\mathrm{K}_{3} \mathrm{CD}_{3}\right)+1 / 2 \mathrm{~V}_{\mathrm{AO}}} \\
& \mathrm{V}_{\mathrm{AF}}=\underset{\text { (voie père-fille) }}{1 / 4 \mathrm{~V}_{\mathrm{AM}}\left(1+\mathrm{K}_{2} \mathrm{CD}_{2}\right)}+\underset{\text { (voie mère-fille) }}{1 / 4 \mathrm{~V}_{\mathrm{AF}}\left(1+\mathrm{K}_{4} \mathrm{CD}_{4}\right)+1 / 2 \mathrm{~V}_{\mathrm{AO}}}
\end{aligned}
$$

En ce qui concerne les pères, on a :

avec :

$$
\mathrm{CD}_{1}=\mathrm{CD}_{2}=\mathrm{n} /\left[\mathrm{n}-1+4\left(\mathrm{~V}_{\mathrm{AF}}+\mathrm{V}_{\mathrm{E}}\right) / \mathrm{V}_{\mathrm{AM}}\right]
$$

$\mathrm{n}=$ nombre de filles de testage par taureau.

$\mathrm{V}_{\mathrm{E}}=$ constante $=$ variance des effets de milieu $=0,75$. La variance unité considérée dans la programmation des calculs est la variance phénotypique qui serait obtenue avec ces mêmes effets de milieu et un coefficient d'héritabilité de 0,25 .

En ce qui concerne les mères, on suppose que l'index de sélection représente la moyenne de 1 lactations, où 1 représente le nombre moyen de lactations $(1=4,6$ sur la voie mère-fils et $1=3,6$ sur la voie mère-fille). Il s'agit d'une approximation puisque en fait les mères sont choisies sur un index combinant les performances, l'index du père et celui de la mère (Poutous et al., 1980).

On a alors :

$$
\mathrm{CD}=\left(\mathrm{V}_{\mathrm{AF}} /\left(\mathrm{V}_{\mathrm{AF}}+\mathrm{V}_{\mathrm{E}}\right)\right) /\left[1+(1-1)\left(\mathrm{V}_{\mathrm{AF}}+\mathrm{V}_{\mathbf{E A}}\right) /\left(\mathrm{V}_{\mathrm{AF}}+\mathrm{V}_{\mathbf{E}}\right)\right]
$$

avec :

$\dot{\mathrm{V}}_{\mathrm{EA}}=$ variance des effets milieu commun à toutes les lactations d'un même animal $=0,25$.

Les valeurs de $\mathrm{K}$ correspondent à des pressions de sélection de 0,$03 ; 0,13 ; 0,003$ et 0,70 , respectivement. 
c) Schémas de transfert $A, B, B^{\prime}$

A l'équilibre, on a :

$$
\begin{aligned}
\mathrm{V}_{\mathrm{AM}}=\begin{array}{l}
1 / 4 \mathrm{~V}_{\mathrm{AM}}\left(1+\mathrm{K}_{1} \mathrm{CD}_{1}\right) \\
\text { (voie père-fils) }
\end{array} & \begin{array}{l}
1 / 4 \mathrm{~V}_{\mathrm{AF}}\left(1+\mathrm{K}_{3}^{\prime} \mathrm{CD}_{3}^{\prime}\right)+1 / 2 \mathrm{~V}_{\mathrm{AO}} \\
\text { (voie mère-fils) }
\end{array} \\
\mathrm{V}_{\mathrm{AF}}=\begin{array}{l}
1 / 4 \mathrm{~V}_{\mathrm{AM}}\left(1+\mathrm{K}_{2} \mathrm{CD}_{2}\right)+ \\
\text { (voie père-fille) }
\end{array} & \begin{array}{l}
1 / 4 \mathrm{~V}_{\mathrm{AF}}\left(1+\mathrm{K}_{4}^{\prime} \mathrm{CD}_{4}^{\prime}\right)+1 / 2 \mathrm{~V}_{\mathrm{AO}} \\
\text { (voie mère-fille) }
\end{array}
\end{aligned}
$$

On a :

$$
\mathrm{CD}_{3}^{\prime}=\mathrm{CD}_{4}^{\prime}=\mathrm{V}_{\mathrm{AF}} /\left(\mathrm{V}_{\mathrm{AF}}+\mathrm{V}_{\mathrm{E}}\right)
$$

d) Schémas de transfert JJ16 et JJ32

$$
\mathrm{V}_{\mathrm{AM}}=\mathrm{V}_{\mathrm{AF}}=\mathrm{V}_{\mathrm{A}}=1 / 4 \mathrm{~V}_{\mathrm{A}}\left(1+\mathrm{K}_{\mathrm{M}} \mathrm{CD}\right)+1 / 4 \mathrm{~V}_{\mathrm{A}}\left(1+\mathrm{K}_{\mathrm{F}} \mathrm{CD}\right)+1 / 2 \mathrm{~V}_{\mathrm{AO}}
$$

où $\mathrm{K}_{\mathrm{M}}$ et $\mathrm{K}_{\mathrm{F}}$ correspondent aux pressions de sélection effectuées sur l'index généalogique et où $\mathrm{CD}$ désigne le coefficient de détermination de cet indice.

\section{Liste des notations}

c Rapport $N^{*} / \mathrm{N}$.

$\mathrm{CD}_{\mathrm{v}} \quad$ Coefficient de détermination de l'index utilisé pour la voie $\mathrm{v}$.

$D_{i} \quad$ Nombre total de descendants du groupe $i$ de pères.

$\Delta \mathrm{F} \quad$ Accroissement annuel de $\mathrm{F}$.

$\Delta \mathrm{G} \quad$ Progrès génétique annuel.

f Nombre de femelles par génération.

$\mathbf{f}_{\mathbf{a}} \quad$ Nombre annuel de nouvelles femelles sélectionnées.

F Coefficient de consanguinité.

$\mathrm{F}_{\mathrm{g}} \quad$ Coefficient de consanguinité à la génération $\mathrm{g}$.

$\mathrm{h}^{2} \quad$ Coefficient d'héritabilité.

h $\sqrt{\mathrm{h}^{2}}$.

$\mathrm{i}_{41}, \mathrm{i}_{42} \quad$ Intensités de sélection correspondant à $\mathrm{p}_{41}, \mathrm{p}_{42}$.

$\mathrm{I}_{\mathrm{v}} \quad$ Supériorité génétique des parents de la voie $\mathrm{v}$ (exprimés en unité d'écarttype génétique).

$\mathrm{k}_{1} \quad$ Nombre de génisses de transfert par femelle donneuse.

$k_{2} \quad$ Nombre de génisses normales par femelle donneuse.

$\mathbf{K}_{\mathbf{v}} \quad$ Coefficient de réduction de variance pour la voie $\mathrm{v}=-\mathbf{i}_{\mathbf{v}}\left(\mathbf{i}_{\mathbf{v}}-\mathbf{x}_{\mathbf{v}}\right)$.

$L_{v} \quad$ Intervalle de génération le long de la voie $v$.

m Nombre de mâles par génération.

$\mathrm{m}_{\mathrm{a}} \quad$ Nombre annuel de nouveaux mâles sélectionnés.

$\mathrm{n}_{1} \quad$ Nombre de femelles de renouvellement du noyau issue de transfert.

$\mathrm{n}_{2} \quad$ Nombre de femelles normales de renouvellement du noyau.

$\mathrm{N}$ Taille du noyau $\left(=\mathrm{n}_{1}+\mathrm{n}_{2}\right)$. 
$\mathrm{N}^{*} \quad$ Nombre de mères potentielles.

$\mathrm{N}_{\mathrm{e}} \quad$ Effectif génétique efficace.

$\mathrm{p}_{41}, \mathrm{p}_{42}$ Taux de conservation effectué sur les mères des génisses de renouvellement issues de transfert ou sur les mères des génisses normales de renouvellement.

$\mathrm{p}_{41}^{*} \quad$ Rapport $\mathrm{n}_{1} / \mathrm{N}$.

$T_{i} \quad$ Nombre total de pères du groupe $i$.

$\mathrm{v} \quad$ Indice voie de transmission du progrès génétique :
$1=$ voie père-fils
$3=$ voie mère-fils
$2=$ voie père-fille
41 = voie mère-fille de transfert
$4=$ voie mère-fille
$42=$ voie mère-fille normale.

$\mathrm{V}_{\mathbf{A F}} \quad$ Variance génétique pour les femelles.

$V_{A M} \quad$ Variance génétique pour les mâles.

$\mathrm{V}_{\mathrm{AO}} \quad$ Variance génétique initiale.

$V_{\mathbf{E}} \quad$ Variance de milieu.

$\mathbf{x}_{41}, \mathbf{x}_{42}$ Valeurs de la variable normale réduite au point de troncature correspondant à $\mathrm{p}_{41}, \mathrm{p}_{42}$.

Reçu le 23 mai 1984.

Accepté le 2 mai 1985.

\section{Références bibliographiques}

ANONYME, 1982. Résultats de contrôle laitier. (France 1982). Fédération nationale des organismes de contrôle laitier. 96 pp., Institut technique de l'élevage bovin. Paris.

AUran T., 1976. Studies on monthly and cumulative milk yield records. III. Estimates of genetic and phenotypic parameters. Acta Agric. Scand., 26, 3-9.

Bonarti B., MocQuot J.C., 1982. Etudes sur la production laitière des bovins. IV - Paramètres génétiques en première lactation. Ann. Génét. Sél. Anim., 14, 161-176.

Bulmer M.G., 1971. The effect of selection on genetic variability. Am. Nat., 105, 201-211.

BURRows P.M., 1972. Expected selection differentials for directional selection. Biometrics, 28, $1091-1100$.

Burrows P.M., 1984. Inbreeding under selection from unrelated families. Biometrics, 40, 357-366.

Chupin D., Procureur R., 1983. Aspects techniques de la transplantation embryonnaire : le traitement des donneuses. Bull. Tech. Insém. Artif., 29, 2-9.

Church R.B., ShEa B.F., 1977. The role of embryo transfer in cattle improvement programs. Can. J. Anim. Sci., 57, 33-44.

Colleau J.J., Poutous M., 1973. Méthode de calcul des index de production laitière des femelles en France. Ann. Génét. Sél. Anim., 5, 73-82.

Cunningham E.P., 1976. The use of egg transfer techniques in genetic improvement. E.E.C. Seminar, Cambridge, 10-12 December 1975. Rowson L.E.A. (ed.), 345-354, E.E.C. Luxembourg.

Danell B., 1981. Studies on lactation yield and individual test-day yields of Swedish dairy cows. II. Genetic parameters. Acta Agric. Scand., 32, 83-91. 
DEMPFLE L., 1975. A note on increasing the limit of selection through selection within families. Genet. Res. Camb., 24, 127-135.

Ducroce V., 1984. Conséquences sur le progrès génétique laitier d'une sélection sur des caractères secondaires chez les bovins. Génét. Sél. Evol., 16, 467-490.

ELSEN J.M., MocQuot J.C., 1976. Intérêt de la transplantation d'œufs pour accroître l'efficacité de la sélection des bovins à viande. Ann. Génét. Sél. Anim., 8, 331-342.

ELSEN J.M., 1980. Diffusion du progrès génétique dans les populations avec générations imbriquées : quelques propriétés d'un modèle de prévision. Ann. Génét. Sél. Anim., 12, 49-80.

Foulley J.L., Chevalet C., 1981. Méthode de prise en compte de la consanguinité dans un modèle simple de simulation des performances. Ann. Génét. Sél. Anim., 13, 189-195.

Hansen L.B., Freeman A.E., Berger P.J., 1983. Association of heifer fertility with cow fertility and yield in dairy cattle. J. Dairy Sci., 66, 306-314.

Heyman Y., Menissier F., Bouyssou B., Nibart M., 1983. Bilan de l'analyse de la base de données constituée par le fichier national des donneuses et des receveuses. (Bilan du programme national pour l'étude et le développement de la transplantation embryonnaire en France). Institut National de la Recherche Agronomique. Union Nationale des Coopératives d'Elevage et d'Insémination, $110 \mathrm{pp}$. Polycopié.

HiLl W.G., 1974. Prediction and evaluation of response to selection with overlapping generations. Anim. Prod., 18, 117-139.

Hill W.G., 1976. Order statistics of correlated variables and implications in genetic selection programmes. Biometrics, 32, 389-902.

HILL W.G., 1977. Order statistics of correlated variables and implications in genetic selection programmes. II. Response to selection. Biometrics, 33, 703-712.

HIL. W.G., 1979. A note on effective population size with overlapping generations. Genetics, 92, 317-322.

HILL W.G., 1984. Effects of population size on rates and variability of response to short and long term selection. 35th Annual Meeting of E.A.A.P., The Hague, Nederlands, 6-9 August 1984, Commission on animal genetics. Mimeo $16 \mathrm{pp}$.

Hill W.G., RoberTson A., 1968. Linkage disequilibrium in finite populations. Theor. Appl. Genet., 33, 226-231.

Hinks C.J.M., 1978. The use of centralised breeding schemes in dairy cattle improvement. Anim. Breed. Abstr., 46, 291-297.

J AMES J.W., 1977. Open nucleus breeding systems. Anim. Prod., 24, 287-305.

JAmes J.W., 1978. Effective population size in open nucleus breeding schemes. Acta. Agric. Scand., 28, 387-392.

JANDRAIN M., 1977. Les répercussions génétiques de la transplantation ovulaire. II - Le cas d'une population de bovins laitiers. Ann. Med. Vet., 121, 175-192.

JANSON L., 1980. Studies on fertility traits in Swedish dairy cattle. II - Genetic parameters. Acta Agric. Scand., 30, 427-436.

Kemeny J.G., Snell J.L., 1976. Finite Markov Chains, 210 pp. Springer Verlag, New York.

KraussLich H., 1976. Application of superovulation and egg transplantation in A.I. breeding programms for dual purpose cattle. E.E.C. Seminar, Cambridge, 10-12 December 1975. Rowson L.E.A. (ed.), 333-344, E.E.C., Luxembourg.

LAND R.B., HILl W.G., 1975. The possible use of superovulation and embryo transfer in cattle to increase response to selection. Anim. Prod., 21, 1-12.

Li C.C., 1955. Population genetics, 366 pp., The university of Chicago Press, Chicago.

Maijala K., Hanna M., 1974. Reliable phenotypic and genetic parameters in dairy cattle. 1 st World Congress on Genetics applied to Livestock production, Madrid, 7-11 October 1974, 1, 541-563, Editorial Garsi, Madrid.

MC Daniel R.T., Cassel B.G., 1981. Effects of embryo transfer on genetic change in dairy cattle. J. Dairy Sci., 64, 2484-2492.

MocQuot J.C., 1982. Impact possible du transfert d'embryons sur l'amélioration génétique les bovins par sélection. Elev. Insém., 191, 11-23. 
Mueller J.P., James J.W., 1983. Effects of reduced variance due to selection in open nucleus breeding systems. Aust. J. Agric. Res., 34, 53-62.

Nicholas F.W., 1979. The genetic implications of multiple ovulation and embryo transfer in small dairy herds. 30th Annual Meeting of the E.A.A.P., 23-26 July 1979, Harrogate (England).

Nicholas F.W., Smith C., 1983. Increased rates of genetic change in dairy cattle by embryo transfer and splitting. Anim. Prod., 36, 341-353.

Онта T., Kimura M., 1969. Linkage disequilibrium due to random genetic drift. Genet. Res. Camb., 13, 47-55.

Fetersen P.H., Hansen M., 1977. Breeding aspects of embryo transplantation utilized in the bull-dam path within a dual purpose cattle population. Livest. Prod. Sci., 4, 305-312.

Pourous M., Briend M., Calomiti S., Doan D., Felgines C., Steier G., 1981. Méthode de calcul des index laitiers. Bull. Tech. Ing. Serv. Agric., 361, 433-446.

Rendel J.M., Robertson A., 1950. Estimation of genetic gain in milk yield by selection in a close herd of dairy cattle. J. Genet., 50, 1-8.

Robertson A., 1961. Inbreeding in artificial selection programmes. Genet. Res. Camb., 2, 189-194.

Robertson A., 1977. Artificial selection with a large number of linked loci. Proceedings of the International Conference in quantitative genetics, 16-21 August 1976, Pollak E., Kempthorn O., Bailey T.B.Jr. (ed.), 307-322, Iowa State University Press, Ames.

SORENSEN D.A., KenNedy B.W., 1983. The use of the relationship matrix to account for genetic drift variance in the analysis of genetic experiments. Theor. Appl. Genet., 66, 217-220.

VAN VLECK L.D., 1977. Theoretical and actual genetic progress in dairy cattle. Proceedings of the International Conference on Quantitative Genetics, 16-21 August 1976, Pollak E., Kempthorn O., Bailey T.B.Jr. (ed.), 543-567, The Iowa State University Press, Ames.

VAN VleCK L.D., 1981. Potential genetic impact of artificial insemination, sex selection, embryo transfer, cloning and selfing in dairy cattle. In BRACKETT B.G., SEIDEL Jr.G.E., SEIDEL S. (ed.), New technologies in animal breeding, 221-242. Academic Press, New York. 